\title{
Spectral Models of Advection\#dominated Accretion Flows with Winds
}

\section{Citation}

Quataert, Eliot, and Ramesh Narayan. 1999. "Spectral Models of Advection\#dominated Accretion Flows with Winds." The Astrophysical Journal 520 (1): 298-315. https://doi.org/10.1086/307439.

\section{Permanent link}

http://nrs.harvard.edu/urn-3:HUL.InstRepos:41384878

\section{Terms of Use}

This article was downloaded from Harvard University's DASH repository, and is made available under the terms and conditions applicable to Other Posted Material, as set forth at http:// nrs.harvard.edu/urn-3:HUL.InstRepos:dash.current.terms-of-use\#LAA

\section{Share Your Story}

The Harvard community has made this article openly available.

Please share how this access benefits you. Submit a story.

\section{Accessibility}




\title{
Spectral Models of Advection-Dominated Accretion Flows with Winds
}

\author{
Eliot Quataert! and Ramesh Narayan? \\ Harvard-Smithsonian Center for Astrophysics, 60 Garden St., Cambridge, MA 02138
}

\begin{abstract}
We calculate spectral models of advection-dominated accretion flows, taking into account the possibility that significant mass may be lost to a wind. We apply the models to the soft X-ray transient V404 Cyg in quiescence and the Galactic center source Sgr A*. We show that there are qualitative degeneracies between the mass loss rate in the wind and parameters characterizing the microphysics of the accretion flow; of particular importance is $\delta$, the fraction of the turbulent energy which heats the electrons. For small $\delta$, current observations of soft X-ray transients and Sgr $A^{*}$ suggest that at least $\sim 10 \%$ of the mass originating at large radii must reach the central object. For large $\delta \sim 0.3$, however, models with significantly more mass loss are in agreement with the observations. We also discuss constraints on advection-dominated accretion flow models imposed by recent radio observations of NGC 4649 and other nearby elliptical galaxies. We conclude by highlighting future observations which may clarify the importance of mass loss in sub-Eddington accretion flows.

Subject headings: accretion, accretion disks - black hole physics
\end{abstract}

\section{Introduction}

A number of authors have argued that, at sub-Eddington accretion rates, the gravitational potential energy released by turbulent stresses in an accretion flow may be stored as thermal energy, rather than being radiated (Ichimaru 1977; Rees et al. 1982; Narayan \& Yi 1994, 1995; Abramowicz et al. 1995; Chen et al. 1995; see Narayan, Mahadevan, \& Quataert 1998b, and Kato, Fukue, \& Mineshige 1998 for reviews). Narayan \& Yi (1994,1995) noted that such advection-dominated accretion flows (ADAFs) have the interesting property that their Bernoulli parameter, a measure of the sum of the kinetic energy, gravitational potential energy, and enthalpy, is positive; since, in the absence of viscosity, the Bernoulli parameter is conserved on streamlines, the gas can, in principle, escape to "infinity" with positive energy. Narayan \& Yi speculated that this might make ADAFs a natural candidate for launching the outflows/jets seen to originate from a number of accretion systems.

Blandford \& Begelman (1998; hereafter BB98) have recently suggested that mass loss via winds in ADAFs may be both dynamically crucial and quite substantial. They construct self-similar ADAF solutions in which the mass accretion rate in the flow varies with radius

\footnotetext{
${ }^{1}$ equataert@cfa.harvard.edu

${ }^{2}$ rnarayan@cfa.harvard.edu
} 
$R$ as $\dot{M} \propto R^{p}$. If the wind carries away roughly the specific angular momentum and energy appropriate to the radius from which it is launched, they show that the remaining (accreting) gas has a negative Bernoulli parameter only for large values of $p \sim 1$. They therefore propose that the majority of the mass originating at large radii is lost to a wind. For example, for $p=1$, only a fraction $\sim\left(R_{\text {in }} / R_{\text {out }}\right) \ll 1$ of the mass would accrete onto the central object, where $R_{\text {in }}$ and $R_{\text {out }}$ are the inner and outer radii of the ADAF.

In a separate study, Di Matteo et al. (1998; hereafter D98) measured the flux of radio and submillimeter emission from the nuclei of nearby elliptical galaxies and found fluxes significantly below the values predicted by the ADAF model. Their observations are difficult to reconcile with Fabian \& Rees's (1995) proposal that these galactic nuclei contain ADAFs. D98 discuss a number of explanations for the "missing" flux; one of their suggestions is that a significant wind may carry off much of the accreting mass in the ADAF.

Spectral models of ADAFs without mass loss have been applied to a number of low luminosity accreting black hole systems. They give a satisfying description of the spectral characteristics of several quiescent black hole binaries (Narayan, McClintock, \& Yi 1996, Narayan, Barret, \& McClintock 1997; Hameury et al. 1997) and low luminosity galactic nuclei, e.g., Sgr A* (Narayan, Mahadevan, \& Yi 1995; Manmoto et al. 1997; Narayan et al. 1998) and NGC 4258 (Lasota et al. 1996a, Gammie, Narayan, \& Blandford 1998).

Our goal in this paper is to use broad-band spectral observations to test for the presence of mass loss in low luminosity accreting black holes, paying special attention to the implications of uncertainties in the microphysics of the accretion flow. Specifically, we attempt to answer the following question: are the no-mass loss ADAF models in the literature, which fit the observations reasonably well, unique "ADAF" fits to the data, or are models with substantial mass loss also viable? If the latter, since it is unlikely that purely theoretical arguments will be definitive, can we distinguish between no-wind and wind models with future observations? As a first step toward addressing these questions, we calculate spectral models of ADAFs with $\dot{M} \propto R^{p}$, and compare them with observations of the X-ray binary V404 Cyg in quiescence, the Galactic center source Sgr A*, and the nucleus of the elliptical galaxy NGC 4649. We assume throughout that all observed radiation from the systems under consideration is due to the accretion flow, i.e., the wind/outflow does not radiate significantly.

In the next section $(\S 2)$, we discuss our modeling techniques. We then show models for V404 Cyg (§3) and Sgr A* $(\S 4)$ and compare the models to observations, focusing on the available theoretical parameter space. In $\S 5$ we discuss D98's results on the radio emission in nearby ellipticals. We then propose several future observations which may help clarify the physics of ADAFs ( $\S 6)$. Finally, in $\S 7$ we summarize and discuss our results.

\section{Modeling Techniques}

Over the last few years, ADAF models have seen a series of improvements such that the modeling techniques used currently are much superior to earlier methods. The first published

spectral models of ADAFs used the self-similar solution of Narayan \& Yi (1994) to model the 
dynamics, but this was soon replaced by global models, initially for a pseudo-Newtonian potential (Narayan, Kato, \& Honma 1997, Chen, Abramowicz, \& Lasota 1997), and more recently in the full Kerr metric (Abramowicz et al. 1996, Peitz \& Appl 1997, Gammie \& Popham 1998). The spectral modeling too has seen improvements, particularly in the treatment of the electron energy equation and the Comptonization. The electron energy equation was originally taken to be local (e.g., Narayan, McClintock, \& Yi 1996), with heating due to Coulomb collisions and turbulent heating balancing cooling. As emphasized by Nakamura et al. (1997), however, the electron entropy gradient (electron advection) generally cannot be neglected, and so this is now included (see eq. [1]).

Narayan et al. (1998a) discuss how the predicted spectra have changed as the modeling techniques have improved. The changes have generally been fairly modest, at least compared to the large changes we see in the present paper when we include mass loss from the accretion flow.

In this paper, we use the latest techniques for numerically calculating spectral models of ADAFs (plus any thin disk at large radii), as described in detail by Narayan et al. (1998a; see also Esin, McClintock, \& Narayan 1998 and Narayan, Barret, \& McClintock 1997). Here we mention only the relevant differences.

As in Narayan et al. (1998a) and Esin et al. (1997), we solve the full electron energy equation, including the electron entropy gradient. The equation takes the form

$$
n_{e} v \frac{d}{d R}\left(\frac{k T_{e}}{\gamma_{e}-1}\right)=k T_{e} v \frac{d n_{e}}{d R}+H_{e}+q_{i e}-q_{e}^{-},
$$

where $T_{e}$ is the electron temperature, $\gamma_{e}$ is the adiabatic index of the electrons, $n_{e}$ is the electron number density, $v$ is the radial velocity, $H_{e}$ is the turbulent heating rate of the electrons, $q_{i e}$ is the energy transferred to the electrons from the ions by Coulomb collisions, and $q_{e}^{-}$is the radiative cooling rate of the electrons. The first term on the right hand side of equation (1) describes the increase in the electron internal energy due to $P d V$ work, and is the volumetric version of $q_{c}$ defined in equation (6) below.

A difference in this paper, relative to earlier work, is that we take $\gamma_{e}$ to be that of a monatomic ideal gas $(5 / 3$ in the non-relativistic limit, decreasing to $4 / 3$ in the relativistic limit). Esin et al. (1998) argued that $\gamma_{e}$ should include contributions from the magnetic energy density in the flow. As discussed in Quataert \& Narayan (1998; their Appendix A), this is incorrect if MHD adequately describes the accretion flow. This is of some significance for models of low luminosity systems. For example, in the "standard" ADAF model of Sgr $\mathrm{A}^{*}$, the electrons are, to good approximation, adiabatically compressed. The larger $\gamma_{e}$ used in this paper yields higher electron temperatures (by a factor of $\sim 3$ ) and significantly more synchrotron emission. As a result, to produce a radio flux comparable to that in Narayan et al. (1998a), we require a noticeably weaker magnetic field.

We describe the turbulent heating of the electrons via a parameter $\delta$, defined by $H_{e} \equiv \delta q^{+}$, where $q^{+}$is the usual "viscous" dissipation rate of accretion theory (e.g., Kato et al. 1998). Thus, $\delta$ is the fraction of the total energy generated by turbulent stresses in the fluid $\left(q^{+}\right)$that directly heats the electrons. As discussed in Quataert \& Narayan (1998), there is a subtlety in interpreting $q^{+}$in ADAFs which is not present in thin disks; namely, only a fraction $\eta(\sim 1 / 2)$ 
of $q^{+}$is likely to end up in the particles; the rest is used to build up the magnetic field and turbulence as the accreting gas flows in. Of the fraction $\eta$, a fraction $\delta_{H}$ goes into electrons and $\left(1-\delta_{H}\right)$ goes into ions. Thus, in terms of $\eta$ and $\delta_{H}$, the $\delta$ we use in this paper is $\delta \equiv \delta_{H} \eta$.

Accounting for a variable mass accretion rate in the flow, the continuity equation becomes

$$
\dot{M}=-4 \pi R^{2} H_{\theta} \rho v=\dot{M}_{\text {out }}\left(\frac{R}{R_{\text {out }}}\right)^{p}
$$

where $H_{\theta}, \rho$, and $v$, are, respectively, the angular scale height, mass density, and radial velocity in the flow. The quantity $\dot{M}_{\text {out }}$ is the accretion rate at the radius $R_{\text {out }}$, where winds become important. We take the radial velocity, angular velocity, and sound speed of the flow from the global, relativistic, models of Gammie \& Popham (1998), and then use equation (2) to calculate the density, $\rho$. This is, strictly speaking, inconsistent, as Gammie \& Popham's models were derived under the assumption of constant $\dot{M}$. The error made in this approximation should, however, be of order unity. From a spectral modeling point of view, the primary importance of the wind is that it modifies the density in the flow; this is correctly captured by equation (2).

Generically, ADAFs with winds will rotate more quickly than those without winds. This is seen in the self-similar solution of BB98, where the rotational support enables the enthalpy of the gas to decrease, thus permitting the Bernoulli parameter to become negative. The shear and the viscous dissipation per unit mass in the flow are therefore expected to be larger in the presence of a wind.2] We have crudely accounted for this effect as follows.

In non-wind models, the flow structure is, among other things, a function of $\gamma_{g}$, the adiabatic index of the fluid. In calculating models of systems with winds and high $\delta$ (Figures $2 \mathrm{~b}, 4 \mathrm{~b}, 7, \& 8)$, we have chosen $\gamma_{g}$ such that it yields a rotation rate in the interior of the flow which is comparable to that expected from the self-similar wind solution of BB98. In particular, a self-similar, non-relativistic, ADAF has a Bernoulli parameter equal to zero only if $\Omega / \Omega_{K} \approx[2 p /(p+5 / 2)]^{1 / 2}$. For our typical value of $p=0.4$, this yields $\Omega / \Omega_{K} \approx 0.53$. In this case we take $\gamma_{g} \approx 1.5$ in our global calculations, since it reproduces this rotation rate well. Note that it is important to get the right $\Omega$ only for high $\delta$. For low $\delta$, since turbulent heating of electrons is unimportant, the exact $\gamma_{g}$ we use is not important. We have confirmed this by calculating models with various choices of $\gamma_{g}$ at low $\delta$ and making sure that the spectral models are only weakly modified.

We are reasonably confident that, even though we have used an ad hoc prescription in choosing the global solutions, our parameter estimates are fairly accurate. Ultimately, of course, global, relativistic, models of ADAFs with winds will be needed to correctly assess some of the issues addressed in this paper.

\footnotetext{
${ }^{1}$ Essentially, the parameter $\eta$ reflects the fact that, just as one must account for advection by the particles, one must also account for advection by the turbulence.

${ }^{2}$ This is actually true only for certain "types" of winds (in particular, depending on BB98's parameters $\epsilon$ and $\lambda$ ). As discussed in, e.g., Blandford \& Payne (1982), it is possible for a wind to take away all of the angular momentum and energy flux from the disk, leaving it cold and dissipationless.
} 


\subsection{Choice of parameters}

We measure black hole masses in solar units and (radially varying) accretion rates in Eddington units: $M=m M_{\odot}$ and $\dot{M}=\dot{m} \dot{M}_{e d d}$. We take $\dot{M}_{e d d}=10 L_{e d d} / c^{2}=2.2 \times$ $10^{-8} m M_{\odot} \mathrm{yr}^{-1}$, i.e., with a canonical $10 \%$ efficiency. We measure radii in the flow in Schwarzschild units: $R=r R_{s}$, where $R_{s}=2 G M / c^{2}$ is the Schwarzschild radius of the black hole.

The parameters of our models are $m, \dot{m}_{\text {out }}=\dot{M}_{\text {out }} / \dot{M}_{\text {edd }}, \beta, \alpha, \delta, r_{\text {out }}$, and $p$. Our primary focus is to consider the effects of winds via the parameter $p$ (defined in eq. [2]). As we show, however, variations in $p$ are qualitatively degenerate with variations in other parameters of the problem.

The mass of the central black hole, $m$, is estimated from observations. As in all previous work, we fix $\dot{m}_{\text {out }}$ by adjusting it so that the X-ray flux in the model fits the available data. For all of the models presented here, $r_{\text {out }}=10^{4}$. Note that $r_{\text {out }}$ and $p$ are, roughly speaking, degenerate; what is of primary importance is $r_{\text {out }}^{-p}$, the fraction of the incoming mass accreted onto the central object. Typical values of $p$ considered are $p=0$ (no winds) and $p=0.4$ (moderately strong wind).

The quantities $\beta \equiv P_{\text {gas }} / P_{\text {mag }}, \alpha$, and $\delta$ are microphysical parameters representing the magnetic field strength in the flow, the efficiency of angular momentum transport, and the fraction of the turbulent energy which heats the electrons, respectively. ? $^{3}$ Recent ADAF models in the literature have favored the values $\beta=1, \alpha=0.25$, and $\delta=10^{-3}$ (cf. Narayan et al. 1998a), and have considered only factor of few variations in $\alpha$ and $\beta$ and factor of $\sim 10$ variations in $\delta$. There is, however, considerable uncertainty in the microphysics of ADAFs; each of the above parameters must be regarded as uncertain to at least an order of magnitude, likely more.

As we will show in this paper, mass loss from the accretion flow has a dramatic effect on theoretically predicted spectra. If we were to restrict ourselves to the values of the microphysics parameters given above, significant mass loss would be all but ruled out by the observations. Such a restriction would, however, be an inaccurate reflection of the theoretical uncertainty in the microphysics of the flow. The philosophy adopted in this paper is therefore somewhat different from previous studies. We allow $\alpha, \beta$ and $\delta$ to vary over a much larger range, but one which we believe correctly encompasses the theoretical uncertainties.

For purely theoretical reasons (see below) we take our "canonical" values to be different from those of previous studies, namely, $\beta=10, \alpha=0.1$, and $\delta=10^{-2}$. By canonical we mean (only) that, when one of the parameters is varied (e.g., $p$ ), the others (e.g., $\delta$ ) are typically fixed at their canonical values. A major point of this paper will be that, depending on the importance of winds, these values may or may not be consistent with observations.

Theoretical work on particle heating in ADAFs (Gruzinov 1998, Quataert 1998, Quataert

\footnotetext{
${ }^{3}$ Our definition of $\beta$ is that utilized in the plasma physics literature. A number of workers in the accretion literature define a " $\beta$ " via $\beta_{\text {adv }} \equiv P_{\text {gas }} / P_{\text {tot }}$, with $P_{\text {tot }}=P_{\text {gas }}+P_{\text {mag }}$. This is related to our $\beta$ by $\beta_{\text {adv }}=$ $3 \beta /(3 \beta+1)$ or $\beta_{\mathrm{adv}}=\beta /(\beta+1)$, depending on whether one defines the magnetic pressure to be $B^{2} / 24 \pi$ or $B^{2} / 8 \pi$ (as we do here).
} 
\& Gruzinov 1998) and "fluid" models for the evolution of the turbulent energy in an ADAF (Quataert \& Narayan 1998) suggest that subthermal magnetic fields may be likely; we consider $\beta=10$ to be a plausible value. We take, however, a range of $\beta$, from $\beta=1$ (strict equipartition of gas and magnetic pressure) to $\beta=100$ (weak fields).

If the turbulent stresses arise solely from magnetic fields, we expect the viscosity parameter to scale roughly as $\alpha \sim 1 / \beta$ (Hawley, Gammie \& Balbus 1996). We do not always enforce this relation in our models, but sometimes vary $\alpha$ and $\beta$ independently. We consider values of $\alpha$ ranging from 0.03 to 0.3 . We should note, however, that large values of $\alpha \approx 0.25$ are needed in applications of the ADAF model to X-ray binaries such as Nova Muscae 1991, Cyg X-1 and GRO J0422+20 in the low/hard state (Narayan 1996, Esin, McClintock \& Narayan 1997, Esin et al. 1998). If $\alpha$ is much smaller than 0.25 , the maximum accretion rate, $\dot{m}_{\text {crit }}$, up to which the ADAF solution is possible decreases significantly, and the maximum luminosity of the models becomes much smaller than the observed luminosities. We have confirmed that this limit on $\alpha$ is not modified if winds are included in the models.

The value of the parameter $\delta$ is uncertain. Traditional ADAF models have taken $\delta$ to be small $\left(\sim 10^{-3}\right)$ and never considered $\delta \gtrsim 0.03$. A number of studies have been carried out to investigate the heating of protons and electrons in hot plasmas. Quataert (1998) and Gruzinov (1998; see also Blackman 1998, Quataert \& Gruzinov 1998) considered particle heating by MHD turbulence and concluded that $\delta$ might be small so long as $\beta$ is greater than about $\sim 10$. Bisnovatyi-Kogan \& Lovelace (1997; see also Quataert \& Gruzinov 1998), however, argue that magnetic reconnection, and its presumed electron heating, may lead to large values of $\delta \sim 1$. f In this paper, we avoid theoretical prejudice and consider values of $\delta$ ranging from 0 to 0.75 . Since the maximum value of $\delta$ is $\eta$, the fraction of the turbulent energy that goes into the particles, the value $\delta=0.75$ likely corresponds to a situation where electrons are heated much more strongly than ions.

\subsection{Description of Spectra}

In the following sections we compare theoretical spectra of ADAFs, both with and without winds, to observations of low-luminosity systems. In preparation for this we introduce here the main features of the calculated spectra.

Three radiation processes are of importance in ADAF spectra: synchrotron emission, Compton scattering, and bremsstrahlung. Each of these produces distinct and easily recognized features in the spectrum. The relative importance of each mechanism is a function of the temperature and density of the plasma, and thus of the model parameters, $\alpha, \beta, \delta, p, m$, and $\dot{m}$.

Thermal synchrotron emission in ADAFs is invariably self-absorbed and produces a sharply cutoff peak, with a peak frequency that depends on the mass of the black hole: $\nu_{s} \sim 10^{15} \mathrm{~m}^{-1 / 2}$

\footnotetext{
${ }^{4}$ Despite our disagreement with some of Bisnovatyi-Kogan \& Lovelace's arguments (see Blackman 1998), their basic point, that reconnection may be crucial for ADAF models, is nonetheless important.
} 
Hz. The synchrotron peak is in the optical band for stellar-mass black holes, and in the radio for supermassive black holes. Synchrotron emission from different radii in the flow occurs at different frequencies. The peak emission, however, is always from close to the black hole and reflects the properties of the accreting gas near $r \sim 1$.

In spectra of quiescent systems of the sort we discuss in this paper $\left(\dot{m}_{i n} \lesssim 10^{-3}\right)$, and especially in the absence of winds, the synchrotron peak is the most luminous feature in the spectrum. The maximum value of $\nu L_{\nu}$ is given by (Mahadevan 1997)

$$
\nu_{s} L_{\nu, s} \propto B^{3} T_{e}^{7} \propto \beta^{-3 / 2} \dot{m}_{i n}^{3 / 2} T_{e}^{7}
$$

where all quantities should be evaluated at $r \sim 1$ and $\dot{m}_{i n}$ is the accretion rate near $r \sim 1$. Note the very steep dependence on the electron temperature.

In writing equation (3), we have taken $\rho \propto \dot{m}$, but independent of $\alpha$, as is appropriate near the central object. This can be understood by noting that, near the central object, the self-similar scaling $\rho \propto \dot{m} / \alpha$ fails. The dynamics in the synchrotron and Compton emitting regimes is dominated by the presence of a sonic point at $r \sim 3-5$ (Narayan, Kato, \& Honma 1997, Chen et al. 1997, Gammie \& Popham 1998). Near this radius the flow velocity is $\sim$ the sound speed, independent of $\alpha$. By the continuity equation, then, the density in the interior scales as $\rho \propto \dot{m}$, with only a weak dependence on $\alpha$. The density on the outside, however, does scale as $\rho \propto \dot{m} / \alpha$ because, away from the sonic point, self-similarity is reasonably valid.

Compton scattering of synchrotron photons by the hot electrons in the accreting gas produces one, or sometimes two peaks in the spectrum at frequencies higher than the synchrotron peak. The peaks correspond to successive scatterings by the electrons. As with the synchrotron peak, the Compton features are again sensitive to the properties of the gas near the black hole.

The frequency of the first Compton peak $\nu_{c}$ is related to $\nu_{s}$ by the Compton boost factor $A$, which is a function only of the electron temperature

$$
\frac{\nu_{c}}{\nu_{s}}=A=1+4 \theta_{e}+16 \theta_{e}^{2}, \quad \theta_{e}=\frac{k T_{e}}{m_{e} c^{2}}=\frac{T_{e}}{5.9 \times 10^{9} \mathrm{~K}} .
$$

The power in the Compton peak relative to that in the synchrotron peak depends on both $A$ and the optical depth of the flow to electron scattering $(\tau)$

$$
\nu_{c} L_{\nu, c} \approx \nu_{s} L_{\nu, s}\left(\frac{\nu_{c}}{\nu_{s}}\right)^{\alpha_{c}}, \quad \alpha_{c}=1+\frac{\ln \tau}{\ln A} .
$$

The relative power in the synchrotron and Compton peaks therefore provides some information on the density in the inner regions of the flow, and thus on $\dot{m}_{i n}$. Note that for the low luminosity systems considered here, $\tau \ll 1$ and $\alpha_{c}<0$, so that the synchrotron luminosity dominates the Compton luminosity.

Finally, bremsstrahlung emission produces a peak that typically extends from a few to a few hundred keV. In contrast to the other two processes discussed above, this emission arises from all radii in the flow. To see this, consider a self-similar ADAF with a wind, for which $\rho \propto r^{-3 / 2+p}$ and $T_{e} \propto r^{-\epsilon}\left(\epsilon \sim 1\right.$ at large radii). Let the minimum flow temperature be $T_{\text {min }}$ (which occurs at $r_{\text {out }}$ ) and the maximum temperature be $T_{\max }$ (near $r \sim 1$ ). At photon energies 
$\ll k T_{\text {min }}$, the bremsstrahlung emission is given roughly by $\nu L_{\nu} \propto \nu$ (the spectral index is a little different from unity because of the Gaunt factor, which we ignore for simplicity), while for $k T_{\min } \ll h \nu \ll k T_{\max }$, it is $\nu L_{\nu} \propto \nu^{1 / 2-2 p / \epsilon}$. In each case, the emission comes from the largest radius which satisfies $h \nu \sim k T(r)$. In our models, $T_{\min }$ is $\sim\left(10^{12} / r_{\text {out }}\right) \mathrm{K}$; therefore, for $r_{\text {out }}=10^{4}, k T_{\text {min }}$ is $\sim 10 \mathrm{keV}$. For X-ray observations in the range $0.1-10 \mathrm{keV}, h \nu \lesssim k T_{\text {min }}$. By above, $\nu L_{\nu}$ should be roughly proportional to $\nu$, but should flatten beyond about $10 \mathrm{keV}$; $\nu L_{\nu}$ will vary as $\nu^{1 / 2}$ beyond $10 \mathrm{keV}$ if there is no wind $(p=0)$ and it will be flatter or even turn over (in $\nu L_{\nu}$ ) if there is a strong wind (large $p$ ).

In all cases, the hardest emission, at $\gtrsim 100 \mathrm{keV}$, occurs from the inner $r \lesssim 100$, while the softer emission comes from $\sim r_{\text {out }}$ (this is particularly true for $p>0$ ). Observations in the 1-10 keV X-ray band are therefore most sensitive to the outer regions of the ADAF. In these regions, the electron temperature is fairly well-determined since the gas is essentially virial and one-temperature. Therefore, observations of the bremsstrahlung emission at a few keV give direct information on the density of the outer flow, and thereby the accretion rate on the outside $\dot{m}_{\text {out }}$.

In the sources that we consider below, the synchrotron peak is isolated and well observed (in X-ray binaries, the companion must be subtracted out); it occurs in softer bands, either in the optical or radio. The Compton and bremsstrahlung peaks, however, can sometimes be superposed in the X-ray band. In particular, an important consequence of the $m$ dependence of the frequency of the synchrotron peak $\left(\nu_{s}\right)$ is that, without winds, the X-ray spectrum of ADAF models of low luminosity galactic black hole candidates is usually dominated by the first Compton peak. In low luminosity AGN, however, the precise behavior in the X-ray band is sensitive to the details (microphysics, accretion rate) of the model, being a competition between the second Compton peak and bremsstrahlung. This is because the peak synchrotron emission is at substantially lower frequencies and a synchrotron photon must be scattered more than once (or off of hotter electrons) in order to be scattered into the X-ray band; this tends to suppress the importance of Comptonization. Note that bremsstrahlung and Comptonization can be readily distinguished by their different spectral slopes in the X-ray band.

If there is a thin disk outside the ADAF, as in our models of X-ray binaries ( $\S 3$ ), the emission of the disk is seen as a blackbody-like feature in the spectrum. This emission is in the red or near infrared for quiescent X-ray binaries in which the disk is restricted to $r>r_{\text {out }} \sim 10^{4}$.

\subsection{The Effects of Winds on Spectral Models}

Bremsstrahlung emission at $\sim 1-10 \mathrm{keV}$ is rather insensitive to the presence of a wind (i.e., to $p$ ) since it originates in the outer regions of the flow and essentially measures $\dot{m}_{\text {out }}$. At higher energies, $\gtrsim 10 \mathrm{keV}$, however, the bremsstrahlung emission decreases with increasing $p$ $\left(\nu L_{\nu} \propto \nu^{1 / 2-2 p / \epsilon}\right)$ and thus provides a powerful probe of the flow density and the value of the parameter $p$ (see $\S 6.2$ ).

By contrast, the predicted synchrotron emission decreases strongly with increasing $p$. There are two reasons for this. First, increasing $p$ decreases the density of the plasma near 
$r \sim 1$, where the high frequency synchrotron emission originates. This implies a lower gas pressure and hence a weaker magnetic field (for fixed $\beta$ ). Perhaps more importantly, the electron temperature decreases as $p$ increases. For the low luminosity systems considered in this paper, and for small $\delta$, the electrons are nearly adiabatic, i.e., $T_{e} \propto \rho^{\gamma_{e}-1} \propto r^{(-1.5+p)\left(\gamma_{e}-1\right)}$. When $p$ is large, the density profile is flatter, adiabatic compression is less efficient, and hence $T_{e}$ is smaller. By equation (3), the synchrotron emission is particularly sensitive to the electron temperature. Therefore, the synchrotron emission falls very rapidly with increasing $p$. This effect can, as we show explicitly below, be countered by increasing $\delta$, since a larger $\delta$ means stronger turbulent heating of the electrons and thus larger $T_{e}$.

The Compton power decreases with increasing $p$ even more strongly than the synchrotron does. As equation (5) shows, $\nu_{c} L_{\nu, c}$ depends on both $\nu_{s} L_{\nu, s}$ and $\alpha_{c}$, both of which decrease because of the wind ( $\alpha_{c}$ decreases because $\tau$ and $\theta_{e}$ both decrease). Increasing $\delta$ to restore the synchrotron power also increases the Compton power, as discussed in the following sections.

\section{Soft X-ray Transients in Quiescence}

Soft X-ray transients (SXTs) are mass transfer binaries which occasionally enter a high luminosity, "outburst," phase, but most of the time remain in a very low luminosity, "quiescent," phase. The spectra of quiescent SXTs are not consistent with a thin accretion disk model, which is unable to account for the fluxes and spectral slopes in the optical and X-ray bands consistently (e.g., McClintock et al. 1995). Narayan, McClintock, \& Yi (1996; see also Narayan et al. 1997a, Hameury et al. 1997) showed that this problem can be resolved if quiescent SXTs accrete primarily via ADAFs, with the thin disk confined to large radii, $r>r_{\text {out }} \sim 10^{4}$. (Note that $r_{\text {out }}$ is taken here to be the same as the transition radius, $r_{t r}$, defined in previous papers. However, it need not be if winds only become important well inside the outer boundary of the ADAF.)

In this section, we give a detailed description of models of the SXT V404 Cyg in quiescence. The X-ray data on V404 Cyg (Narayan et al. 1997a) are much superior to the data on other SXTs, which makes this system better suited for the parameter study we present.

Table 1 lists the various parameter combinations we have tried for modeling V404 Cyg, and some of the characteristics of these models, including the microphysical parameters, the maximum electron temperature, and the radiative efficiency. Following Shahbaz et al. (1994), we have taken the mass of the black hole to be $m=12$.

Outbursts in SXTs are believed to be triggered by a thermal-viscous instability in the thin disk (enhanced mass transfer from the companion may also be important). Initial ADAF models of black hole SXTs in quiescence (Narayan, McClintock, \& Yi 1996) assumed that the observed optical emission from these systems was blackbody emission from a steady state outer thin disk. Wheeler (1996) and Lasota, Narayan, \& Yi (1996) pointed out that this was inconsistent because quiescent thin disks are not likely to be in steady state. Furthermore, in non-steady quiescent disks, the mass accretion rate decreases rapidly with radius (so as to maintain a roughly constant effective temperature $\sim$ a few thousand K; e.g., Cannizzo 1993). 
This implies a limit on $r_{\text {out }}$; if $r_{\text {out }}$ is too small, then the disk cannot supply sufficient mass to the inner ADAF to fit the X-ray observations. Quantitatively, the limit is $r_{\text {out }} \gtrsim 10^{4}$ for V404 Cyg (it is slightly smaller for A0620-00). We fix $r_{\text {out }}=10^{4}$ in all the models presented here, and we take the thin disk to extend from $r=10^{4}$ to $10^{5}$.

\subsection{Spectral Models of V404 Cyg}

Figure 1a shows spectral models of V404 Cyg for different $p$ for our standard microphysics parameters: $\alpha=0.1, \beta=10$, and $\delta=0.01$. We see two important effects of changing $p$.

First, in the X-ray band, models with weak winds (small $p$ ) have Compton-dominated $\mathrm{X}$-ray spectra, while models with strong winds (large $p$ ) are bremsstrahlung dominated. The reason for this has already been explained in $\S 2$. The Compton emission comes from near the black hole, while the bremsstrahlung comes from the outer regions of the ADAF. As the wind becomes stronger, the inner mass accretion rate $\dot{m}_{\text {in }}=\dot{m}_{\text {out }} r_{\text {out }}^{-p}$ becomes significantly smaller than $\dot{m}_{\text {out }}$, reducing the importance of Comptonization relative to bremsstrahlung.

Associated with this switch is another interesting feature. For weak winds (small values of $p$ ), we see that $\dot{m}_{i n}$ remains roughly constant when we change $p$ (e.g. $\dot{m}_{i n}=10^{-3}, 9 \times 10^{-4}$, for $p=0,0.2$ ), while for large values of $p$, it is $\dot{m}_{\text {out }}$ that remains roughly constant (e.g. $\dot{m}_{\text {out }}=0.016,0.02$, for $\left.p=0.4,0.6\right)$. This is again easy to understand once we realize that the mass accretion rate is adjusted so as to reproduce the X-ray flux. When the model is Compton-dominated, the X-ray flux depends on $\dot{m}_{i n}$, and so this quantity remains roughly the same as $p$ varies. However, when bremsstrahlung dominates, the X-ray flux depends on $\dot{m}_{\text {out }}$ and so it is $\dot{m}_{\text {out }}$ that remains constant.

The second effect that is seen in Figure 1a (and even more clearly in Fig. 4a for Sgr $\mathrm{A}^{*}$ ) is that the synchrotron emission becomes weaker as the wind becomes stronger. Once $p$ is large enough $(\gtrsim 0.2)$ for the model to become bremsstrahlung-dominated, $\dot{m}_{\text {out }}$ is more or less frozen at a fixed value. For yet larger $p, \dot{m}_{i n}$ decreases rapidly with increasing $p$. Since the synchrotron emission depends primarily on $\dot{m}_{i n}$, the synchrotron peak drops significantly in magnitude. The decrease in the synchrotron power at large $p$ is actually more dramatic than is apparent in Figure 1a (see Fig. 4a). Most of the optical/infrared flux in the $p=0.4,0.6$ models in Figure 1a is blackbody emission from the outer disk, which depends only on $\dot{m}_{\text {out }}$, and does not change with $p$ at large $p$. This emission is cool (it is limited by the disk's effective temperature, which is about $5000 \mathrm{~K}$ ) and the peak occurs at lower frequencies.

The above analysis hinges on the change in the flow density with $p$. How is it modified if the microphysical parameters are varied from the canonical values taken above? Figure 1b shows models with a moderate wind, $p=0.4$, for various values of the parameter $\beta$, which determines the strength of the magnetic field ( $\alpha$ and $\delta$ are fixed at their canonical values of 0.1 and 0.01 , respectively). Changing $\beta$ has little effect in the X-ray band since bremsstrahlung emission does not depend on the magnetic field strength. Increasing $\beta$ to $\sim 1$ naturally increases the synchrotron flux (eq. [3]). Even for $\beta=1$, however, the synchrotron luminosity is too low by a factor of $\sim 2-3$. Note that, for $\beta=1$, the optical emission in the models of Figure $1 \mathrm{~b}$ is 
primarily synchrotron, while for $\beta \gtrsim 10$ it is primarily disk emission.

Figure 2a shows models of V404 Cyg for $p=0.4$ for several $\alpha$ ( $\beta$ and $\delta$ are fixed at their canonical values of 10 and $10^{-2}$ ). These models show little variation in X-ray behavior with $\alpha$, but there is a decrease in optical emission as $\alpha$ decreases. This can be understood as follows. In the self-similar regime (reasonably valid at large radii), the flow density in an ADAF is $\rho \propto \dot{m} / \alpha$. Furthermore, the X-ray flux, which we fix to the observed value, arises from the outer regions of the ADAF via bremsstrahlung. Since the bremsstrahlung luminosity is $\propto \rho^{2}$, $\dot{m}_{\text {out }} / \alpha$ remains roughly constant as $\alpha$ varies $\left(\dot{m}_{\text {out }}=5 \times 10^{-3}, 1.6 \times 10^{-2}, 2.6 \times 10^{-2}\right.$ for $\alpha=0.03,0.1,0.3)$. All three models therefore have nearly the same density and temperature on the outside, which accounts for the lack of significant change in the X-ray band. For these models, however, the optical is dominated by disk emission, which is proportional to $\dot{m}_{\text {out }}$, rather than $\dot{m}_{\text {out }} / \alpha$. For smaller $\alpha, \dot{m}_{\text {out }}$ is smaller and thus the disk emission decreases, as seen in Figure 2a.

Finally, Figure 2b shows models of V404 Cyg for $p=0.4$ for several $\delta(\beta=10, \alpha=0.1)$. For small $\delta \lesssim 10^{-2}$, the electrons are heated primarily by adiabatic compression (the first term on the right in eq. [1]) and so the results are nearly independent of the value of $\delta$. However, once $\delta \gtrsim 10^{-2}$, turbulent heating $\left(H_{e}\right)$ becomes the dominant heating mechanism. In this regime, increasing $\delta$ causes the electrons to become hotter (see Table 1), thereby increasing the synchrotron emission and Comptonization. For sufficiently large $\delta \gtrsim 0.1$, Comptonization dominates bremsstrahlung in the X-ray band, and the spectra begin to resemble the no-wind model shown by the solid line in Figure 1a.

The above results are for ADAF models with winds, since that is the primary focus of this paper. For completeness, we have considered the sensitivity of no-wind (or weak wind) models to variations in $\alpha, \beta$ and $\delta$. Figure 3a shows models of V404 Cyg with $p=0$ taking, for brevity, $\alpha \sim \beta^{-1}$, as suggested by numerical simulations of thin accretion disks (Hawley, Gammie, \& Balbus 1996). We see that larger values of $\alpha$ (and lower $\beta$ ) lead to more synchrotron emission. Figure $3 \mathrm{~b}$ shows models for various $\delta$. Increasing $\delta$ leads to a noticeable increase in the electron temperature. This is seen explicitly in Table 1 and also in the larger "displacement" of the Compton peak relative to the synchrotron peak (see eq. 柾 for the Compton A parameter). Since the synchrotron and Compton emission increase strongly with temperature, the model with the largest $\delta$ has a significantly lower $\dot{m}$ (Table 1 ).

\subsection{Comparison with Observations}

Figures 1-3 show the available observational constraints on the spectrum of V404 Cyg (taken from Narayan et al. 1997a). The optical data give the luminosity of the source and constrain the effective temperature of the radiation to be $\gtrsim 10^{4} \mathrm{~K}$. There is an upper limit on the EUV flux, which is not very interesting since it is easily satisfied by all the models considered here. Thanks to an excellent ASCA observation, the luminosity in the X-ray band is known accurately, and the spectral index is also well constrained; in terms of $\nu L_{\nu} \propto \nu^{2-\Gamma}$, the $2 \sigma$ error bars on the photon index $\Gamma$ are $2.1_{-0.3}^{+0.5}$. 
The observations give a few important constraints. First, the $>10^{4} \mathrm{~K}$ temperature of the optical argues against the outer thin disk as the source of this radiation (Lasota, Narayan, \& Yi 1996b; see below). Thus, the optical has to come from synchrotron and this emission must be stronger than the disk emission. Second, the observed photon index in X-rays in V404 Cyg is incompatible with the $\Gamma=1$ expected for thermal bremsstrahlung ( $(2)$. This means that the X-ray emission has to be Compton-dominated. There is preliminary evidence that the same is also true for A0620-00 (Narayan et al. 1996, 1997a), but the ROSAT data on that source (McClintock et al. 1995) are not sufficiently good to trust this conclusion; on the other hand, for GRO J1655-40, preliminary ASCA data in quiescence indicate a much harder X-ray spectrum than in V404 Cyg and A0620-00 (Hameury et al. 1997). Finally, the data show that the optical emission is about an order of magnitude larger (in $\nu L_{\nu}$ ) than the X-ray flux, another constraint that has to be satisfied by models.

The baseline no-wind ( $p=0)$ model of V404 Cyg, with canonical values for the microphysics parameters, is shown by the solid line in Figure 1a. This model fits the observations well, as emphasized by Narayan et al. (1997a). It has roughly the right luminosity and effective temperature in the optical and is consistent with the X-ray data. The model shown here differs somewhat in the X-ray band from that shown in Narayan et al. (1997a). The difference is due to the different energy equation used here, which leads to hotter electrons and more pronounced Compton bumps. The value of $\dot{m}$ is also lower by a factor of a few.

The observed X-ray spectral index in V404 Cyg places interesting constraints on models. For weak winds $(p \sim 0)$ the models are in agreement with the observed slope for a wide range of microphysical parameters (Figure 3). For strong winds, however, most of the models are too bremsstrahlung-dominated to fit the X-ray slope. For small $\delta \sim 10^{-2}$, the observed slope rules out $p \gtrsim 0.3$, for any $\alpha$ and $\beta$ (Figures 1-2). For the value of $r_{\text {out }}=10^{4}$ we have taken, this means that at least $\sim 10 \%$ of the mass supplied from the companion must reach the central object.

As discussed by Lasota et al. (1996b), a thin disk cannot account for the observed optical emission in quiescent SXTs. This is because thin disk annuli with effective temperatures comparable to the observed values, $\sim 10^{4} \mathrm{~K}$, are thermally and viscously unstable. In fact, within the context of the disk instability model, quiescent disks in black hole SXTs have effective temperatures $\sim 3000-5000 \mathrm{~K}$ (Lasota et al. 1996b), too low to account for the observations. This is an independent argument against high $p$, low $\delta$ ADAF models, since the optical emission in these models is always dominated by the disk (the synchrotron being strongly suppressed by the large $p$ ).

Perhaps the most interesting result to come out of these comparisons is that wind models agree with the data for larger values of the electron heating parameter $\delta$. The $p=0.4, \delta=0.3$ model in Figure $2 \mathrm{~b}$ is as good as the no wind low- $\delta$ model shown in Figure 1a. The increase in $T_{e}$ associated with increasing $\delta$ brings the synchrotron emission into rough agreement with the observed optical flux, despite the low value of $\dot{m}_{i n}$ 抱 at the same time, it shifts the balance

\footnotetext{
${ }^{5}$ The synchrotron peak is a little too cool to fit the data; given the model uncertainties, however, the difference is not large enough to argue against these models.
} 
in the X-ray band from bremsstrahlung to Comptonization, as required by observations.

\section{The Galactic Center}

Observations of the Galactic Center indicate that the mass of the black hole in Sgr A* is $m \sim(2.5 \pm 0.4) \times 10^{6}$ (Haller et al. 1996; Eckart \& Genzel 1997; Genzel et al. 1997). The accretion rate is estimated to lie in the range $10^{-4} \lesssim \dot{m}_{\text {out }} \lesssim$ few $\times 10^{-3}$ (Genzel et al. 1994; Melia 1992), with the upper end of the range considered more likely (Coker \& Melia 1997). For a radiative efficiency of $10 \%$, and assuming that $\dot{m}$ is constant in the accretion flow, the implied luminosity is between $\sim 10^{40} \mathrm{erg} \mathrm{s}^{-1}$ and $\sim 10^{42} \mathrm{erg} \mathrm{s}^{-1}$. This is well above the bolometric luminosity of $\lesssim 10^{37} \mathrm{erg} \mathrm{s}^{-1}$ inferred from observations in the radio to $\gamma$-rays (see Narayan et al. 1998a for a review of the observations).

An optically thin, two temperature, ADAF model is a possible explanation for the low luminosity of Sgr A* (Rees 1982; Narayan, Yi, \& Mahadevan 1995, Manmoto et al. 1997, Narayan et al. 1998a, Mahadevan 1998). An alternative explanation is that most of the gas supplied at large radii is lost to a wind and very little reaches the central black hole (BB98). We consider both possibilities in this section.

There is little observational evidence in $\mathrm{Sgr} \mathrm{A}^{*}$ for (or against) a particular value of $r_{\text {out }}$. In addition, there is little evidence that the accretion outside $r_{\text {out }}$ occurs via a thin disk. In our models, we set $r_{\text {out }}=10^{4}$ and assume that, whatever form the plasma takes at larger radii, it is non-radiating.

\subsection{Spectral Models of Sgr $\mathbf{A}^{*}$}

The parameters of each of our models of Sgr A* are given in Table 2.

Figure 4a shows spectral models of Sgr $\mathrm{A}^{*}$ for various $p$, taking $\alpha=0.1, \beta=10$, and $\delta=0.01$. As usual, the value of $\dot{m}_{\text {out }}$ in each model has been adjusted to fit the X-ray flux (even though the ROSAT measurement used in the fits is really only an upper limit; cf. Narayan et al. 1998a). The results in Figure 4a are similar to those shown in Figure 1a for V404 Cyg, but the effects are somewhat more pronounced.

At $\sim 1 \mathrm{keV}$, the baseline no-wind $(p=0)$ model in Figure 4 a corresponds to an interesting situation: there are roughly equal contributions from the second Compton bump and bremsstrahlung. Recall that increasing $p$ always shifts the balance in favor of bremsstrahlung. Therefore, once $p$ is increased above zero, the Compton flux decreases, and the spectrum becomes bremsstrahlung-dominated in the X-ray band. This switch is evident already at $p=0.2$ and it becomes more pronounced for larger $p$. The three bremsstrahlung-dominated models with $p=0.2,0.4$ and 0.6 all have nearly the same value of $\dot{m}_{\text {out }} \approx 2 \times 10^{-4}$, while there is a modest change in $\dot{m}_{\text {out }}$ between $p=0$ and 0.2 (see Table 2 ).

Another effect seen very clearly in Figure 4a is the decrease in the synchrotron emission in the radio with increasing $p$. This is due to a decrease in both the magnetic field strength 
and $T_{e}(\S 2)$. Note, in particular, that $T_{e}$ decreases by a factor of $\approx 5$ from $p=0$ to $p=0.6$ (Table 2).

The dependence of wind models of Sgr $\mathrm{A}^{*}$ on the microphysical parameters is very similar to that of V404 Cyg. The one exception is that all of the $p \gtrsim 0.2$ models in Figure 4-6 are bremsstrahlung-dominated in X-rays; we practically never see Comptonized power in the Xray band. This is simply because the source of soft photons - the synchrotron peak - is at substantially lower frequencies in Sgr A* compared to the SXTs (recall that $\nu_{s} \propto m^{-1 / 2} ; \S 2.2$ ).

Figures 4b, 5a and 5b show models of Sgr $\mathrm{A}^{*}$ for $p=0.4$ and different values of $\beta, \alpha$ and $\delta$. We reach two conclusions from these calculations. First, no combination of $\alpha$ and $\beta$ alone is sufficient to bring the synchrotron emission of wind models back to the level seen in the baseline no-wind model (Figures 4b \& 5a). Just as in V404 Cyg, however, increasing $\delta$ has a very strong effect on the synchrotron emission. Indeed, a $p=0.4, \delta=0.3$ model has roughly the same synchrotron power as the $p=0, \delta=0.01$ no-wind model. The reason is clear increasing $\delta$ causes a substantial increase in $T_{e}$ (Table 2), which compensates for the reduced density and field strength due to the wind. By contrast, neither $\alpha$ nor $\beta$ has a comparable effect.

As Figure 5a shows, decreasing $\alpha$ decreases the radio emission in Sgr $\mathrm{A}^{*}$. This is because, near the central object, $\rho \propto \dot{m}$, and is only a weak function of $\alpha$. The density on the outside, however, scales as $\rho \propto \dot{m} / \alpha$. If we fix the X-ray flux, $\dot{m}_{\text {out }}$ has to decrease as $\alpha$ decreases in order to keep $\rho$ the same on the outside and thereby produce the same level of bremsstrahlung radiation. This causes a decrease in the density in the interior of the flow and thus a decrease in the synchrotron emission in the radio (Figure 5a). Small values of $\alpha$ therefore add to the decrease in synchrotron emission that is associated with a strong wind.

Finally, Figure 6 shows models of Sgr A* with no winds $(p=0)$ for several $\alpha \sim \beta^{-1}$ (Fig. $6 a)$ and for several $\delta$ (Fig. 6b).

\subsection{Comparison with Observations}

Figures 4-6 show the observational data on Sgr A*. The source has been reliably detected only in the radio and mm bands, where there is a good spectrum available (see Narayan et al. 1998a for original references to the data). It has been convincingly demonstrated that there is a break in the radio spectrum at around $50-100 \mathrm{GHz}$, so that the source apparently has two components, one which produces the emission below the break and the other above (Serabyn et al. 1997, Falcke et al. 1998). The latter component, which cuts off steeply somewhere between $10^{12}$ and $10^{13} \mathrm{~Hz}$, has been fitted with the ADAF model (Narayan et al. 1998a). The model does not, however, fit the low frequency radio emission. This emission may be from an outflow (e.g. Falcke 1996), or, as in the model of Mahadevan (1998), may be due to non-thermal electrons (in Mahadevan's model, these, along with positrons, are created by the decay of charged pions created in proton-proton collisions). In the following we consider a model to be satisfactory if it fits the high frequency radio data.

In the infrared, Menten et al. (1997) obtained a conservative 2.2 micron flux limit of 9 mJy, 
after accounting for extinction. The source may, however, be variable, since in later epochs Genzel et al. (1997) observed a $K \sim 15$ source at the location of Sgr A*; this corresponds to $F_{\nu} \approx 13$ mJy (Andreas Eckart, private communication). If verified, this would suggest that the infrared flux varies around a mean value of order a few $\mathrm{mJy}$. This is a potentially stringent constraint on theoretical models. We, however, adopt a more conservative approach and treat the IR data as an upper limit. The implications of an IR detection are discussed in $\S 6$.

Although we fit our models to the ROSAT X-ray observations of the galactic center, they too should be treated as an upper limit because of ROSAT's poor angular resolution $(\approx 20 ")$ and the presence of diffuse emission at the Galactic Center. This is again the conservative approach, since a decrease in the X-ray flux would necessitate a decrease in the importance of mass loss; see $\S 6$.

Vargas et al. (1998) have recently provided new SIGMA upper limits on hard X-ray emission from the Galactic Center: between $40-75 \mathrm{keV}$ the luminosity is $\lesssim 1.4 \times 10^{35}$ ergs $\mathrm{s}^{-1}$ while between $75-150 \mathrm{keV}$ it is $\lesssim 2.0 \times 10^{35} \mathrm{ergs} \mathrm{s}^{-1}$. We have converted these to limits in $\nu L_{\nu}$ by assuming that the spectrum is flat in $L_{\nu}$, as would be appropriate for a no-wind bremsstrahlung spectrum.

The solid line in Figure 4a (and the dotted line in Figure 6) shows our standard, nowind $(p=0)$, model, with $\beta=10, \alpha=0.1$, and $\delta=0.01$. Figure 6 shows no-wind models for a number of other microphysics parameters. All of the no-wind models are in reasonable agreement with the data. In particular, they explain the mm fluxes fairly well as synchrotron emission, and produce Compton emission in the infrared roughly consistent with the Menten et al. (1997) limit. Relatively lower $\delta$, larger $\beta$, and smaller $\alpha$ are favored if the IR limit is taken to be stringent; if, however, the Genzel et al. (1997) observations are interpreted as a detection, the opposite is true - larger $\delta$ and/or smaller $\beta$ are favored. In addition, the small $\beta$, large $\delta$ models tend to slightly overproduce the synchrotron emission at $\sim 10^{12} \mathrm{GHz}$.

Note that these conclusions are somewhat different from those of Narayan et al. (1998a), who advocated strict equipartition $(\beta=1)$. As discussed in $\S 2$, this is due to our use of a monatomic ideal gas adiabatic index in the electron energy equation. For small $\delta$, the electrons in Sgr A* are nearly adiabatic; since our adiabatic index is larger than that of Narayan et al. the electrons are hotter in our models. This accounts for the increased synchrotron emission and the need for weaker fields (larger $\beta$ ) for a fixed radio flux. To obtain a radio flux comparable to Narayan et al's $\beta=1$ model, we require $\beta \approx 30$ for $p=0$, or else $p \approx 0.2$. In fact, our no-wind, low $\delta$, models of Sgr A* are rather similar to those of Manmoto et al. (1997), who noted that smaller $\alpha$ were favored if the IR limit in Sgr $\mathrm{A}^{*}$ is taken to be stringent. This is because our treatment of the electron energy equation is similar to Manmoto et al.'s. They took the electron adiabatic index to be $\gamma_{e}=5 / 3$, which is correct in not including a magnetic contribution. ] $^{-1}$

What about large $p$, dynamically important winds? Such winds decrease the density and electron temperature in the interior of the flow, thereby severely suppressing the synchrotron

\footnotetext{
${ }^{6}$ It is incorrect, however, in neglecting the change to $\gamma_{e} \approx 4 / 3$ for $r \lesssim 10^{2}$ when the electrons become semi-relativistic.
} 
and Compton emission (Figure 4a). Requiring wind models to produce the observed $10^{11}-10^{12}$ $\mathrm{Hz}$ emission imposes the following strong constraints on the parameters.

For small $\delta$, we require $p \lesssim 0.2$ if we allow $\beta \sim 1, \alpha \sim 0.3$. If, for theoretical reasons, we were to favor larger $\beta \sim 10-100$, the constraint is even stronger. For the value of $r_{\text {out }}=10^{4}$ used in our models, this corresponds to at least $15 \%$ of the mass supplied at large radii reaching the central object. As in V404 Cyg, the strongest degeneracy in the problem is with $\delta$. For $\delta \gtrsim 0.3$, large $p$ models of Sgr $\mathrm{A}^{*}$ are in good agreement with the data (Figure $5 \mathrm{~b}$ ).

All ADAF models of Sgr A* in the literature have $\dot{m}_{\text {out }} \sim 10^{-4}$. This is at the lower end of the values considered plausible from Bondi capture of stellar winds in the Galactic center, and may be $\sim 10-100$ times smaller than favored values (Coker \& Melia 1997). It is interesting to see that winds do not alter this conclusion (see Table 2). Neither wind nor non-wind models can have $\dot{m}_{\text {out }}$ much greater than $\sim 10^{-4}$ because, if they did, the bremsstrahlung emission would yield an X-ray luminosity well above the observed limits. Since the bremsstrahlung emission at $\sim 1 \mathrm{keV}$ is from the largest radii in the accretion flow, this conclusion is independent of the strength of winds in the system.

In this context, it is important to note that, although $p=0$, large $\delta$ models produce spectra reasonably consistent with the observations (Fig. 6b), they require small accretion rates, $\dot{m}_{\text {out }} \sim 10^{-5}$ (Table 2 ). This argues against them as viable models.

\section{Nuclei of Nearby Ellipticals}

D98 recently measured high frequency radio fluxes from the nuclei of several nearby giant elliptical galaxies. These galactic nuclei are known to be unusually dim in X-rays compared to the accretion rates inferred from Bondi capture (Fabian \& Canizares 1988). Fabian \& Rees (1995) explained the low X-ray luminosities by invoking accretion via ADAFs. D98 found, however, that the predicted radio emission, based on the ADAF model (for $\beta=1$ ), exceeded their measured fluxes by $2-3$ orders of magnitude. They suggested several explanations for this large discrepancy, including the presence of strong winds or highly subthermal magnetic fields.

If, as we are inclined to believe is the case, $\operatorname{Sgr} A^{*}$ is simply a scaled version of the systems observed by D98, why is the predicted emission in Sgr A* roughly in accord with the observations while that in the nearby ellipticals is so discrepant? We might expect both theoretical predictions to be wrong, or both to be right, if the same physics operates in each system. We see two potential answers to this question.

One possibility lies in the X-ray constraints in Sgr A* versus those in D98's sample. In Eddington units, i.e, scaled with respect to the mass of the black hole, the X-ray detection/upper limit in $\mathrm{Sgr} \mathrm{A*}$ is $\sim 2.5$ orders of magnitude below the upper limits in D98's sample. This means that we have a significantly stronger constraint on the accretion rate in Sgr $\mathrm{A}^{*}$. If the X-ray luminosities (in Eddington units) of the ellipticals were as low as in Sgr A*, then models similar to those that work for Sgr A* would work for the ellipticals as well. It would mean, however, that D98's estimate of $\dot{m}_{\text {out }}$ is too large, by a factor $\sim 30$ (see below). 
The other possibility is that the high frequency $\left(>10^{11} \mathrm{~Hz}\right)$ radio observations of Sgr $\mathrm{A}^{*}$, which the ADAF model fits reasonably well, do not probe the accretion flow at all. If the high resolution VLBI observations at $86 \mathrm{GHz}$ represent the true synchrotron emission from the ADAF in Sgr $\mathrm{A}^{*}$, and the higher frequency radio emission is from a completely different source, then typical no-wind (e.g. $p=0, \beta \sim 1$ ) models would overpredict the synchrotron luminosity by $\sim 3$ orders of magnitude, just as D98 found for the ellipticals.

To investigate these issues further, Figure 7 shows a series of models of NGC 4649, which D98 consider to be the most convincing member of their sample. The data are taken from their Table 5. We take $m=8 \times 10^{9}$ (slightly higher than D98's $4 \times 10^{9}$ because we find this mass fits the location of the radio peak better), $r_{\text {out }}=10^{4}$, and assume a distance of $15.8 \mathrm{Mpc}$. All calculations were done with $\alpha=0.1$ and $\beta=10$. Table 3 shows the parameters for the models.

The solid line in Figure 7a corresponds to our "standard" ADAF model: $p=0, \delta=0.01$, and $\dot{m}_{\text {out }}=10^{-3}$. The latter value corresponds to the Bondi mass accretion rate estimated by D98. In agreement with D98, we find that, at this accretion rate, the model overpredicts the radio emission by $\sim 3$ orders of magnitude. To make matters worse, our model is also in violation of the X-ray upper limit, in contrast to D98, whose "standard" ADAF model just satisfies the upper limits. The difference is primarily because our electrons are hotter - D98 used Esin et al's (1998) electron adiabatic index.

We have varied $p$ and $\dot{m}_{\text {out }}$ in our models to judge their sensitivity to these parameters. The dotted line in Figure 7a is a model with $p=0.25$ and $\delta=10^{-2}$. This is roughly the $p$ we need to account for the observed radio flux at low $\delta$ (note that this model is also in agreement with the X-ray upper limit).

In Figure $7 \mathrm{~b}$ we show several models of NGC 4649 for $\dot{m}_{\text {out }}=10^{-4.5}$. This accretion rate is $\sim 30$ times smaller than the value D98 infer from Bondi capture. The solid line shows a standard no-wind model: $p=0$ and $\delta=0.01$. This model is in reasonable agreement with the radio flux. If $T_{e}$ and $\beta$ are fixed, equation (3) shows that the peak synchrotron luminosity scales like $\nu L_{\nu} \propto \dot{m}^{3 / 2}$. Thus, to decrease $\nu L_{\nu}$ by a factor of $10^{3}$, as required by the observations, $\dot{m}_{\text {out }}$ must decrease by $\sim 100$. In fact, due to other factors, the required decrease is even less, $\sim 30$.

The above argument requires that $T_{e}(r)$ should be roughly the same for $\dot{m}_{\text {out }}=10^{-3}$ and for $\dot{m}_{\text {out }}=10^{-4.5}$. This is confirmed by the numerical results shown in Table 3 , but it can also be understood simply by noting that in both models the electrons adiabatically compress as the gas flows in. To see this, it is sufficient to estimate the PdV energy gained per unit time

\footnotetext{
${ }^{7}$ Initially, we found important quantitative differences between our models with varying $p$ and $\dot{m}_{\text {out }}$ and the models in the original version of D98's paper on astro-ph. We have determined, however, that this was due to the fact that they did not use the electron temperature profile appropriate for the given $\dot{m}_{\text {out }}$ and $p$ (Di Matteo, private communication). In particular, they originally required $p=1$ and $r_{\text {out }}=300$ to fit the radio flux at $\dot{m}_{\text {out }}=10^{-3}$, while their new calculations give $p \approx 0.8$ and $r_{\text {out }} \approx 80$ (since they take the inner radius of the flow to be $r=3$, this corresponds to $\approx 7 \%$ of the incoming mass accreted, comparable to our value of $\approx 10 \%$ ). In addition, at $p=0$, they originally required $\dot{m}_{\text {out }}=10^{-6}$ to fit the radio flux, while they now require $\dot{m}_{\text {out }} \approx 10^{-5}$, again in reasonable agreement with our value of $\dot{m}_{\text {out }} \approx 10^{-4.5}$.
} 
by the electrons in a spherical shell of radius $R$ and thickness $d R \sim R$ as they accrete onto the central object (cf eq. [1]),

$$
q_{c} \approx k T_{e} v \frac{d n_{e}}{d R} 4 \pi R^{3} \approx \frac{m_{e}}{m_{p}} \theta_{e}(r) \dot{M}(r) c^{2} \approx 10^{43}\left(\frac{\theta_{e}}{1}\right)\left(\frac{\dot{m}}{10^{-3}}\right)\left(\frac{m}{8 \times 10^{9}}\right) \operatorname{ergs~s}^{-1} .
$$

Our most luminous model (solid line, $\dot{m}_{\text {out }}=10^{-3}$; Figure $7 \mathrm{a}$ ) has $\nu L_{\nu} \approx 10^{41.5} \mathrm{ergs} \mathrm{s}^{-1}$ at the synchrotron peak (and a bolometric luminosity of $\approx 10^{42} \mathrm{ergs} \mathrm{s}^{-1}$ ), which is $\ll q_{c}$. For lower $\dot{m}_{\text {out }}$, the ratio of $\nu_{s} L_{\nu, s}$ to $q_{c}$ is even smaller. Therefore, the electrons in all of our low $\delta$ models are nearly adiabatic, and thus $T_{e}(r)$ is essentially unchanged as $\dot{m}_{\text {out }}$ decreases from $10^{-3}$ to $10^{-4.5}$.

The short dashed lines in Figure 7 show $p=0.25, \delta=0.3$ models for $\dot{m}_{\text {out }}=10^{-3}$ (Figure 7a) and $\dot{m}_{\text {out }}=10^{-4.5}$ (Figure 7b). As suggested by the previous results on V404 Cyg and Sgr $\mathrm{A}^{*}$, these models are comparable to the $p=0, \delta=0.01$ models. In particular, for

$\dot{m}_{\text {out }}=10^{-4.5}$, the wind model gives reasonably good agreement with D98's radio data, while for $\dot{m}_{\text {out }}=10^{-3}$ it is in disagreement.

The results of Figure 7 thus lead to two scenarios for understanding NGC 4649, depending on which value of $\dot{m}_{\text {out }}$ we take, $10^{-4.5}$ or $10^{-3}$. (There is, of course, a range of intermediate scenarios if we take intermediate values of $\dot{m}_{\text {out }}$.)

If $\dot{m}_{\text {out }} \approx 10^{-4.5}$, then we require $0 \lesssim p \lesssim 0.25$ for $0 \lesssim \delta \lesssim 0.3$. As we saw for V404 Cyg and Sgr $\mathrm{A}^{*}$, increasing $p$ requires a corresponding increase in $\delta$, though the precise mapping between the two parameters in the case of NGC 4649 is slightly different. As in V404 Cyg and Sgr $A^{*}$, strong wind, low $\delta$ models are ruled out as they cannot explain the radio data (dotted line; Figure 7b).

If, on the other hand, $\dot{m}_{\text {out }} \approx 10^{-3}$, as proposed by D98, then we require $0.25 \lesssim p \lesssim 0.55$ for $0 \lesssim \delta \lesssim 0.3$. Low $\delta$, low $p$, is ruled out by the observed radio flux (Figure 7a), which is a different result from that obtained in V404 Cyg and Sgr A*. In addition, the region of $p-\delta$ space available for NGC 4649 at $\dot{m}_{\text {out }}=10^{-3}$, if applied to our models of V404 Cyg and $\operatorname{Sgr} \mathrm{A}^{*}$, is somewhat uncomfortable. For example, at $p \approx 0.25$ and low $\delta$ (which gives an acceptable fit in NGC 4649 if $\dot{m}_{\text {out }} \approx 10^{-3}$ ), the predicted X-ray spectral index in V404 Cyg is only marginally compatible with the $2 \sigma$ ASCA measurements (Figure 1a). Similarly, the radio luminosity of Sgr $\mathrm{A}^{*}$ for $p=0.25$ and $\delta=0.01$ is $1-2$ orders of magnitudes below the peak observed luminosity. One might therefore have to abandon the claim that the $10^{11}-10^{12}$ $\mathrm{Hz}$ emission in $\mathrm{Sgr} \mathrm{A}^{*}$ is synchrotron emission from the ADAF.

If we believe that Sgr A*, V404 Cyg, and NGC 4649 are simply scaled versions of each other (in $m$ and $\dot{m}_{\text {out }}$, and perhaps somewhat in $p, \delta, \beta, \alpha$, and $r_{\text {out }}$ ), the above considerations are suggestive, if only weakly, of an $\dot{m}_{\text {out }} \sim 10^{-4.5}$ rather than $10^{-3}$ in NGC 4649 . This conclusion is independent of the importance of winds.

\section{Key Future Observations}

There are two main conclusions from the previous sections: (i) If the electron heating parameter $\delta$ is small, current observations rule out ADAF models with moderate winds (say 
$p \gtrsim 0.25$ as an average for V404 Cyg and Sgr $\mathrm{A}^{*}$ ). (ii) If $\delta$ is allowed to have large values

- given the uncertain role of magnetic reconnection there is no strong theoretical argument against this - current observations provide no information on the importance of winds in ADAFs; large $\delta$, strong wind models are in as good agreement with the data as low $\delta$, weak wind models.

Figure 8 shows the $p / \delta$ degeneracy explicitly for V404 Cyg (Fig. 8a) and Sgr A* (Fig. $8 \mathrm{~b})$. We see that the two $p=0.4, \delta \approx 0.3$ models are very similar to the $p=0, \delta=0.01$ models. Indeed, there is a family of intermediate solutions with values of $p$ and $\delta$ in between these two extremes. Note, however, that the very large $p=0.8, \delta=0.75$ models shown in Figure 8 differ more noticeably. For such large $p$, the electron temperatures needed to make the X-ray spectrum of V404 Cyg Compton dominated, rather than bremsstrahlung dominated, are so large that the Compton peak moves well into the X-ray band. This is discussed further in the next subsection. In the case of $\operatorname{Sgr} A^{*}$, for $p \sim 1, r_{\text {out }} \sim 10^{4}$ and $\dot{m}_{\text {out }} \sim 10^{-4}$, the inner mass accretion rate is $\dot{m}_{i n} \sim 10^{-8}$. The density in the interior is then so low that the synchrotron emission is no longer highly self-absorbed; this accounts for the substantially broader synchrotron peak.

Leaving aside the $p=0.8$ models, we conclude that there is a degeneracy between $p$ and $\delta$ such that any model in the range $0<p \lesssim 0.5$ and $0<\delta \lesssim 0.4$ is viable, so long as $p$ and $\delta$ are chosen in some proportionate manner. Current observations are insufficient to break this degeneracy and additional observational tests are clearly needed to improve the situation. Below we suggest a number of such tests, some of which will be feasible in the near future (e.g., AXAF; $\S 6.3-\S 6.5)$, while others will require a more concerted observational effort (e.g., $\S 6.1 \& \S 6.2)$.

\subsection{Position of the Compton Peak}

Generically, strong wind models have a lower density and optical depth than weak wind models. If these models are to fit the synchrotron and X-ray flux, and have a Comptondominated X-ray spectrum (as required in SXTs for instance), they must have a larger $T_{e}$. The larger $T_{e}$ is necessary to boost the synchrotron luminosity, and also to reproduce the required $\alpha_{c}$ (cf eq. [5]), despite the smaller optical depth. A larger $T_{e}$ implies a larger amplification factor $A$. Thus, the "distance" between the synchrotron and Compton peaks in large $\delta$ wind dominated systems will generally be larger by a factor of a few than in weak wind systems. This is seen explicitly in Figure 8a. Note in particular the $p=0.8, \delta=0.75$ model, where $A$ is so large that the synchrotron peak has moved substantially to the left and at the same time the peak of the Compton emission is well into the X-ray band. This is, however, not a unique property of wind models, but rather is characteristic of any model with a very high $T_{e}$, as can be seen by the $\delta=0.3, p=0$ model in Figure 3b.

In principle, the Compton A parameter could be measured in SXTs, with observations in the optical and soft X-ray $(\sim 0.1 \mathrm{keV})$ bands. The strong Galactic absorption below a $\mathrm{keV}$, however, makes direct detection of the Compton peak problematic, except in very high $\delta$ models. A more encouraging possibility is that the peak's position could be inferred by 
detection of curvature at $\sim 1 \mathrm{keV}$. This should be feasible with AXAF or XMM (§6.4).

\subsection{Shape of the Bremsstrahlung Spectrum}

Detailed measurements of the bremsstrahlung spectrum of an ADAF system can explicitly probe the density profile and outer radius of the accretion flow. This constitutes one of the most direct tests for the presence of winds.

As discussed in $\S 2$, at photon energies $\gtrsim$ the minimum electron thermal energy in the flow, which is a function of the outer radius, bremsstrahlung should give rise to a $\nu L_{\nu} \propto \nu^{1 / 2-2 p / \epsilon}$ spectrum (where $\epsilon \approx 1$; see $§ 2.2$ ). This behavior is clearly seen in Figures 1a (V404 Cyg), 4a $\left(\operatorname{Sgr} A^{*}\right)$, and, in particular, Figure $8 \mathrm{~b}\left(\operatorname{Sgr} A^{*}\right)$, where the bremsstrahlung emission cuts off strongly at $\gtrsim 10 \mathrm{keV}$ for large $p$. More importantly, the details of the cutoff, e.g., the slope at $\sim 10 \mathrm{keV}$, are a strong function of $p$, thus providing the opportunity to study winds directly through their effect on the density profile of the flow. While energies $\gtrsim 10 \mathrm{keV}$ are somewhat high for observations of quiescent systems with current X-ray detectors, they may still be observationally accessible. Sgr $\mathrm{A}^{*}$ is an excellent source in which to apply this technique since a bremsstrahlung-dominated X-ray spectrum is expected above a few $\mathrm{keV}$ for a wide range of the microphysical parameters. The SXTs are probably less useful, since the Compton peak is generally more important.

One potential complication is that, if winds only become important well inside the outer boundary of the flow (for which, at least within the context of BB98's proposal, we see no obvious theoretical reason), the bremsstrahlung emitting region will be unaffected by the wind. Non-detection of a strong $\sim 10 \mathrm{keV}$ bremsstrahlung cutoff therefore would not rule out winds, although it would place interesting constraints on the radius at which mass loss becomes important.

\subsection{Observations of SXTs in Quiescence}

Measurements of the X-ray spectral index in SXTs by AXAF and, in particular, XMM (with its larger collecting area) will clearly be important. Bremsstrahlung predicts a photon spectral index of $\Gamma \sim 1$, while Comptonization predicts a less hard spectrum.

ASCA observations rule out $\Gamma \sim 1$ in V404 Cyg; therefore, models in which Comptonization dominates are favored (Narayan et al. 1997a). Confirmation of this in additional systems would be of considerable interest. Note that detection of a bremsstrahlung spectrum would all but rule out weak wind $(p \approx 0)$ models of quiescent SXTs. As Figure 3 shows, for no combination of $\alpha, \beta$ and $\delta$ can such a spectrum be produced.

When Comptonization dominates, the $0.5-10 \mathrm{keV}$ X-ray band is often the energy range where the first and second Compton bumps meet. Therefore, most models predict significant curvature, i.e., an energy dependent spectral index, within the band. The spectrum would be softer at lower photon energies and harder at higher energies. Detection of curvature of 
this sign would be an important confirmation of the Compton origin of the X-ray emission. Curvature of the opposite sign will be seen only if $\delta$, and therefore the Compton $A$-parameter, is so large that the Compton peak moves into the middle of the X-ray band ( $\S 6.1)$.

It is equally important to measure the optical/UV spectrum of quiescent SXTs. An unambiguous determination that the temperature of the optical radiation is above the maximum temperature $\sim 5000 \mathrm{~K}$ of the outer thin disk (Lasota et al. 1996), would immediately suggest that the optical emission is synchrotron emission from the ADAF. This information would be very useful, since only a subset of models will be able to fit both the observed synchrotron power and the position of the peak.

Although significantly tighter observational constraints on the energy-dependent spectral index in X-rays and the synchrotron peak in the visible/UV would narrow the available theoretical parameter space, it will be difficult to infer unique model parameters from these observations. There is simply too much degeneracy in the models. In particular, strong wind, large $\delta$ models of SXTs can readily produce X-ray and optical behavior similar (though not identical) to that of weak wind models (see Figure 8a). The variations introduced by $\alpha$ and $\beta$ only complicate things further (Figure 3).

\subsection{Observations of Sgr A*}

\subsubsection{X-ray Observations}

Observations of Sgr A* may be particularly helpful in discriminating among theoretical models. An AXAF/XMM detection of Sgr A* will help in two ways. First, a significantly higher resolution detection will determine whether the ROSAT observation was an upper limit. If significantly so, $\dot{m}_{\text {out }}$ will need to decrease in order to fit the reduced X-ray flux; the synchrotron emission in the radio will decrease as well (all other parameters being held fixed). In the case of no winds $(p=0)$, this would argue for stronger magnetic fields and/or larger $\delta$. In wind scenarios, the amount of mass loss in the flow would be further constrained and/or the models would be pushed towards larger $\delta \sim 1$.

As Figures 4-5 and Figure $8 \mathrm{~b}$ show, all of the models of Sgr A* with noticeable winds have a bremsstrahlung dominated X-ray spectrum with $\Gamma \sim 1$. This is true even for the large $\delta$ models which agree with the radio observations (Fig. 8b). Confirmation of this prediction would be of considerable interest. It would not, however, rule out weak wind scenarios, although strictly $p=0$ models would be constrained to having small $\alpha$ and $\delta$ and large $\beta$ (see Figure 6). 8 On the other hand, an observed spectral index deviating significantly from bremsstrahlung, e.g., $\Gamma \gtrsim 2$, would strongly constrain mass loss via winds, arguing for $p \lesssim 0.2$ and/or larger $\delta$.

\footnotetext{
${ }^{8}$ Even at $p=0.2$ (a relatively weak wind in the scheme of things) the X-ray band would be bremsstrahlung dominated for a wide range of microphysical parameters, eliminating these constraints.
} 


\subsubsection{Infrared Observations}

Additional constraints on the accretion in Sgr A* may come from infrared observations. Menten et al. (1997) obtained strong upper limits on the $2.2 \mu \mathrm{m}$ flux from Sgr $\mathrm{A}^{*}$ at an angular resolution of $0.15^{\prime \prime}$, while Genzel et al. (1997) reported a possible infrared detection of Sgr A* at a level above the Menten et al. limit, indicating possible variability in the source.

As seen in Figures 4-6 \& 8b, the 2.2 micron $\mathrm{K}$ band corresponds to the location of the first Compton peak (if present) in ADAF models. If Genzel et al.'s detection is confirmed, and interpreted as the Compton peak, it would argue against significant mass loss due to winds in Sgr $\mathrm{A}^{*}$. As seen in Figures 4, 5, \& 8b, large $p$ models have difficulty, even at large $\delta$, accounting for a Compton luminosity at the level of the Menten et al. (1997) upper limit $(p \lesssim 0.2$ and large $\delta \sim 1$ is tenable). This is because the Compton power decreases more rapidly with $p$ than the synchrotron power. Increasing $\delta$ to bring the synchrotron power back into agreement still leaves the Compton power smaller than in no-wind models (see Fig. 8b).

Equally interesting constraints on models will arise if the infrared limits decrease substantially. This would argue for weak magnetic fields and/or small $\delta$ in no wind scenarios (see Figure 6) or for the presence of a reasonable wind. If the IR limits decrease substantially, it may be difficult for theoretical models to reconcile the absence of a Compton peak (the source of IR emission in the models) with the interpretation of the $\sim 10^{12} \mathrm{~Hz}$ emission as synchrotron emission from the flow.

An alternative to the Compton interpretation of the infrared flux is that it is due to synchrotron emission from non-thermal electrons. Mahadevan (1998) has described a specific model of this kind in which the infrared flux is from electron/positron pairs created by the decay of charged pions in the accretion flow.

\subsubsection{Gamma-ray Observations}

Mahadevan et al. (1997) showed that gamma-ray emission, due to the decay of neutral pions created in proton-proton collisions, may be detectable from an ADAF in Sgr A*. They argued that it may account for the EGRET source 2EG J1746-2852, although current theoretical predictions suggest that the ADAF contribution to these observations may be small (Narayan et al. 1998a, Mahadevan 1998; see Markoff et al. 1997 for an alternative discussion of gamma-ray emission from Sgr A*). Since the gamma-ray luminosity is $\propto \rho^{2}$, the confirmed detection of gamma-rays from $\mathrm{Sgr} \mathrm{A}^{*}$ (i.e., a detection with reasonable angular resolution which shows variability) would directly constrain the density of the plasma and thus the strength of winds. GLAST may provide such observations.

A complication in this analysis is the unknown, and almost certainly non-thermal (Mahadevan \& Quataert 1997, Blackman 1998), proton distribution function. If there is a significant population of relativistic protons at all radii in the flow, the emission will contain important contributions from large radii (Mahadevan et al. 1997), and will thus be a complicated convolution of the density profile of the flow and the (radially varying) proton distribution

function. If, on the other hand, relativistic protons are only present in the interior of the 
flow, e.g., if the protons are heated by incompressible turbulence (Gruzinov \& Quataert 1998), gamma-ray observations would impose particularly strong constraints on the flow properties near $r \sim 1$. Specifically, they will provide a lower limit on the mass accretion rate near the black hole, which, when combined with the estimate of $\dot{m}_{\text {out }}$ obtained from measurements of the bremsstrahlung emission, would strongly constrain the wind parameter $p$.

\subsubsection{Measurements of Radio Brightness Temperatures}

VLBI observations of Sgr A* at $43 \mathrm{GHz}$ and $86 \mathrm{GHz}$ indicate brightness temperatures in excess of $10^{10} \mathrm{~K}$ (Backer et al. 1993, Rogers et al. 1994). This was a problem for Narayan et al's (1998a) no wind model since their electron temperature was everywhere below $10^{10} \mathrm{~K}$. With the revised electron adiabatic index used in this paper $(\S 2)$, we find $T_{e} \gtrsim 10^{10} \mathrm{~K}$ for $r \lesssim 10$, so this is less of a concern.

Small $\delta$, large $p$ models give $T_{e} \lesssim 5 \times 10^{9} \mathrm{~K}$ at all radii in the flow (see Table 2 ), incompatible with the observations. This is because, as noted in $\S 2.3$, large $p$ implies less compression and hence lower $T_{e}$. These low values of $T_{e}$ are an independent argument against high $p$, low $\delta$ models.

For $\delta \gtrsim 0.1$, turbulent heating of the electrons is sufficiently strong that $T_{e}$ is again $\gtrsim 10^{10} \mathrm{~K}$, compatible with the observations. For a fixed radio flux, large $p$ models must have higher electron temperatures to compensate for the lower flow densities; the difference is, however, only a factor of a few. Nonetheless, a careful comparison of theoretical and observed temperature profiles may help constrain the models. If brightness temperatures $\gtrsim 3 \times 10^{10} \mathrm{~K}$ are observed, they would argue for larger $\delta$, in either wind or non-wind scenarios, or for nonthermal electrons.

\subsection{Observations of the Nuclei of Nearby Ellipticals}

As is clear from Figure 7 , one way to resolve the degeneracy between $\dot{m}_{\text {out }} \approx 10^{-3}$ and $\dot{m}_{\text {out }} \approx 10^{-4.5}$ models of NGC 4649 , and the associated $p / \delta$ degeneracy, is through better X-ray limits in this and other nearby ellipticals. The $\dot{m}_{\text {out }} \approx 10^{-3}$ scenario predicts an X-ray flux comparable to the current limits, so AXAF or XMM should be able to detect these systems. On the other hand, the $\dot{m}_{\text {out }} \approx 10^{-4.5}$ scenario predicts X-ray fluxes $\sim 3$ orders of magnitude beneath current limits.

D98's observations in the radio and mm bands with the VLA and SCUBA emphasize the usefulness of such observations for testing ADAF models. They allow both the synchrotron power and the position of the peak to be measured. Additional observations of low luminosity nuclei, including LINERs (Lasota et al. 1996a), will further constrain theoretical models. 


\section{Summary}

The goal of this paper has been to explore spectral models of ADAFs with winds/mass loss, by applying the models to the soft X-ray transient V404 Cyg, the Galactic Center source Sgr $\mathrm{A}^{*}$, and the nucleus of the nearby elliptical galaxy NGC 4649. The first two of these systems are explained fairly well by the ADAF model without any winds. However, recent theoretical arguments (BB98; see also Narayan \& Yi 1994, 1995), as well as observations of NGC 4649 (D98), suggest that mass loss via winds may be important (even dynamically crucial) in subEddington, radiatively inefficient, accretion flows. We have therefore investigated under what conditions ADAF models with mass loss might account for the observations, and the extent to which observations can distinguish between the various proposals for the physics of the accretion flow. A fundamental assumption of our analysis is that, in spite of the possibility of substantial mass loss, the observed radiation is due only to the matter that accretes onto the central object, with no contribution from the outflow.

In assessing the importance of mass loss, considerable care must be taken in how one treats the microphysics of the accretion flow; this is parameterized by: $\alpha$, the viscosity parameter, $\delta$, the fraction of the turbulent energy which heats the electrons, and $\beta$, the ratio of the gas to the magnetic pressure. As we have shown in this paper, mass loss from the accretion flow has a dramatic effect on theoretically predicted spectra. If we were to restrict ourselves to the microphysics parameters considered in previous treatments of ADAFs $(\alpha \sim 0.3, \beta \sim 1$, and $\delta \sim 10^{-3}$ ), significant mass loss would be incompatible with observations. Such a restriction would not, however, correctly reflect the uncertainty in the microphysics of the flow. We have therefore varied $\alpha, \beta$ and $\delta$ over a large range $(\alpha \epsilon[0.03,0.3], \beta \epsilon[1,100]$, and $\delta \epsilon[0,1])$, which we believe generously encompasses the theoretical uncertainties. Despite this large parameter space, firm and interesting constraints on theoretical models can still be drawn.

Spectral models of ADAFs without mass loss provide a reasonable description of the observations of a number of low luminosity black hole systems. In fact, it is this success which has led to the recent wide interest in ADAFs. From our study of spectral models that include mass loss/winds, we reach two principal conclusions. First, if the turbulent heating of the electrons is weak $(\delta \lesssim 0.01$ ), then winds must also be relatively weak ( $p \lesssim 0.25$ ); specifically, the mass accreted by the central black holes in V404 Cyg and Sgr A* must be at least $\sim 10 \%$ of the mass supplied at large radii (for any $\alpha$ and $\beta$ ). Second, for larger values of $\delta$, current observations do not readily assess the importance of winds in ADAFs. Strong wind $(p=0.4)$, large $\delta(\sim 0.3)$ models of V404 Cyg and Sgr $\mathrm{A}^{*}$ are in as good agreement with the data as weak wind $(p \sim 0)$, low $\delta(\lesssim 0.01)$ models; indeed, there is a family of acceptable models in which $p$ and $\delta$ are assigned intermediate values in a proportionate manner.

In $\S 6$, we have proposed a number of observational tests which should help to resolve the $p / \delta$ degeneracy. Radio, infrared, optical/UV, X-ray and gamma-ray observations of quiescent soft X-ray transients, the Galactic Center, and the nuclei of other galaxies provide powerful and complementary information on the various emission processes in the accretion flow and can potentially pin down the importance of winds in ADAFs.

D98's observations of radio emission in nearby ellipticals such as NGC 4649 seem to suggest 
that mass loss may be important; confirmation of this interpretation of their observations, however, requires substantially better X-ray observations than are presently available. Current $\mathrm{X}$-ray upper limits and radio observations alone are compatible with weak (or no) mass loss at accretion rates $\sim 30$ times smaller than the typical Bondi value inferred by D98 ( 55 ). X-ray detection of these systems near current limits would favor high accretion rates and substantial mass loss while noticeably stronger upper limits would favor low accretion rates and weaker mass loss ( $\S 6.5$; Figure 7$)$.

In this context, it is interesting to note that Sgr $\mathrm{A}^{*}$ itself may have a discrepancy between the accretion rate favored by ADAF models and that favored by hydrodynamical simulations of Bondi capture. All non-wind ADAF models in the literature have $\dot{m}_{\text {out }} \sim 10^{-4}$, while Bondi capture estimates are often 10 - 100 times larger (e.g., Coker \& Melia 1997). Winds do not alleviate this discrepancy (see Table 2); neither strong wind nor non-wind ADAF models of Sgr $A^{*}$ can have $\dot{m}_{\text {out }}$ much greater than $\sim 10^{-4}$ because, if they did, the bremsstrahlung emission would yield an X-ray luminosity well above the observed limits.

\subsection{Radiative Efficiency}

Previous discussions of ADAFs in the literature have emphasized the low radiative efficiency of these accretion flows and the connection between the low efficiency and the presence of an event horizon in the central object (Narayan et al. 1997ab, Narayan et al. 1998a, Menou, Quataert \& Narayan 1998). How is this modified when there is a wind?

Tables 1-3 give the radiative efficiencies of our models, defined both with respect to the accretion rate at the outer edge of the flow $\left(\eta_{o} \equiv L / \dot{M}_{\text {out }} c^{2}\right)$ and the accretion rate at the horizon $\left(\eta_{i} \equiv L / \dot{M}_{\text {in }} c^{2}\right)$. The former is perhaps observationally accessible, while the latter is of more physical interest from the point of view of inferring the presence of a horizon. In the absence of winds, $\eta_{o}=\eta_{i} \equiv \eta$.

Qualitatively, a viable spectral model with strong winds weakens the argument for the presence of an event horizon. This is because, in the presence of a strong wind, the central object accretes less mass, but if the microphysical parameters can be adjusted so that the accretion flow produces the same luminosity, then $\eta_{i}$ can be noticeably larger. What remains to be seen is, quantitatively, how $\eta_{i}$ varies with $p$ and the microphysical parameters.

The models of most interest are those in Figure 8, the sequence of roughly similar $p / \delta$ models of V404 Cyg and Sgr A*. The standard no wind models of V404 Cyg and Sgr A* have $\eta \approx 10^{-3}$ and $2.6 \times 10^{-5}$, respectively, which makes both sources highly inefficient radiators. As we increase $p$, and correspondingly increase $\delta$ to keep a roughly similar luminosity, $\eta_{i}$ increases. For the intermediate values of $p=0.4$ and $\delta \sim 0.3, \eta_{i}$ has increased by nearly an order of magnitude for both systems. The radiative efficiency is, however, still $\ll 1$, particularly for Sgr A*.

For the larger values of $p=0.8$ and $\delta=0.75, \eta_{i} \approx 0.1$ for V404 Cyg and $\eta_{i} \approx 0.01$ for Sgr A*. The model of V404 Cyg appears to be incompatible with the observations. The synchrotron peak in the optical has shifted to much lower frequencies in this model and we 
believe that this discrepancy is serious and hard to overcome. Nevertheless, if we are willing to allow models with this level of deviation from the data (there are, after all, uncertainties in our modeling techniques in this extremal range of parameter space), then it means that there are viable ADAF models of V404 Cyg in which the luminosity of the accretion flow is comparable to the rest mass energy accreted by the central object. This weakens the argument for an event horizon in this source.

In the case of $\operatorname{Sgr} \mathrm{A}^{*}$, the $p=0.8, \delta=0.75$ model is in moderate agreement with the data (given some allowance for modeling uncertainties), but it is still a relatively inefficient radiator $\left(\eta_{i} \approx 0.01\right)$. The difference between $\mathrm{Sgr} \mathrm{A}^{*}$ and V404 Cyg in this context is, of course, that $\dot{m}_{\text {out }}$ is quite a bit smaller in Sgr A* (because of the constraints imposed by X-ray observations), so that it naturally has a lower radiative efficiency if all other model parameters are the same. In fact, we find that all of our models of Sgr A* with $\dot{m}_{\text {out }} \sim 10^{-4}$, which fit the observations reasonably well, have $\eta_{i} \lesssim 0.01$. At small $p$, this is because only small values of $\delta$ are allowed if $\dot{m}_{\text {out }} \sim 10^{-4}$ (see Table 2). At large $p$, the density in the interior of the flow is so low that, even if $\delta \equiv 1$ or the plasma is one temperature, the electron cooling time is $\gtrsim$ the inflow time of the gas. As a result, the accretion flow is always reasonably advection dominated, because the electrons themselves are. Therefore, although the argument for the event horizon in Sgr $\mathrm{A}^{*}$ is significantly less dramatic because of the possibility of substantial mass loss $\left(\eta_{i} \approx 0.01\right.$ for $p \approx 0.8$ vs. $\eta_{i} \approx 2.6 \times 10^{-5}$ for $\left.p \approx 0\right)$, it nonetheless appears that all viable ADAF models of Sgr A* have $\eta_{i}$ substantially smaller than the usual thin disk value of $\eta_{i} \approx 0.1$. This problem should be investigated in more detail, in particular, with a better treatment of the dynamics of large $p$ models.

BB98 noted that the presence of winds would cause the difference between the quiescent luminosities of black hole and neutron star soft X-ray transients to reduce. This is discussed further in Menou et al. (1998).

\subsection{Discussion}

In all of our models we have assumed that winds lead to an $\dot{m} \propto r^{p}$ profile inside $r_{\text {out }}=$ $10^{4}$. This corresponds to $10^{-4 p}$ of the incoming mass being accreted. The spectral models are, however, primarily sensitive to the fraction of the mass accreted, rather than to $p$ and $r_{\text {out }}$ separately. If one favors a smaller range of radii over which winds are important, this corresponds roughly to a new $p^{\prime}=4 p / \log r_{w}$, where $r_{w}$ is the radial extent of the region where winds are important. Note, however, that if this approach is taken, our values of $\delta$ may only be upper limits, since a model with a larger $p^{\prime}>p$ will rotate more quickly, and thus have a larger viscous dissipation per unit mass.

In particular, then, our conclusion that low $\delta$ models of Sgr A* and V404 Cyg are compatible with the observations only for $p \lesssim 0.25$ need not conflict with theoretical estimates that $p \sim 1$ is needed for the Bernoulli parameter of the accreting gas to be negative. It may simply mean that winds are important only over $\sim 1-1.5$ decades of radius $\left(r_{w} \approx 10-30\right)$, instead of 4 decades as we have assumed here. Nonetheless, it is clear that for winds to be both dynamically crucial (large $p$ ) and take away the majority of the mass (large $r_{w}$ ), large 
values of $\delta$ are required.

Acknowledgments. We thank Jeff McClintock, Kristen Menou, and Jun-Hui Zhao for useful discussions. Jean-Pierre Lasota provided a number of useful comments on an earlier version of this paper. This work was supported by an NSF Graduate Research Fellowship and by NASA Grant NAG 5-2837. 


\section{References}

Abramowicz, M., Chen, X., Granath, M., \& Lasota, J.P. 1996, ApJ, 471, 762

Abramowicz, M., Chen, X., Kato, S., Lasota, J.-P., \& Regev, O., 1995, ApJ, 438, L37

Backer, D., C, 1982, in Proc. IAU Symposium, eds. D.S. Heeschen \& C. M. Wade, 97, 389

Blackman, E., 1998, MNRAS in press astro-ph/ 9710137)

Blandford, R. D. \& Begelman, M. C., 1998, MNRAS submitted (astro-ph/9809083) (BB98)

Blandford, R. D. \& Payne, D.G., 1982, MNRAS, 199, 883

Bisnovatyi-Kogan, G. S., \& Lovelace R. V. E., 1997, ApJ, 486, L43

Cannizzo, J. K., 1993, in "Accretion Disks in Compact Stellar Systems," ed. J. Wheeler (Singapore: World Scientific), p. 6

Chen, X., Abramowicz, M.A., Lasota, J.-P., Narayan, R., \& Yi, I. 1995, ApJ, 443, L61

Chen, X., Abramowicz, M.A., Lasota, J.-P., 1997, ApJ, 476, 61

Coker, R. \& Melia, F., 1997, ApJ, 488, L149

Di Matteo, T., Fabian, A. C., Rees, M. J., Carilli, C. L., \& Ivison, R. J., 1998, MNRAS, submitted astro-ph/9807245) (D98)

Eckart, A., \& Genzel, R. 1997, MNRAS, 284, 576

Esin, A. A., McClintock, J. E., \& Narayan, R., 1997, ApJ, 489, 86

Esin, A. A., Narayan, R., Cui, W., Grove, E., \& Zhang, S., 1998, ApJ in press (astro-ph/9711167)

Fabian, A. C. \& Canizares, C. R., 1988, Nature, 333, 829

Fabian, A. C. \& Rees, M. J., 1995, MNRAS, 277, L55

Falcke, H., 1996, in IAU Symp. 169, Unsolved Problems of the Milky Way, eds. L. Blitz \& P. J. Teuben (Dordrecht: Kluwer), 163

Falcke, H., Goss, W. M., Matsuo, H., Teuben, P., Zhao, J., \& Zylka, R., 1998, ApJ, 499, 731

Gammie, C.F., Narayan, R., \& Blandford, R., 1998, MNRAS submitted astro-ph/9808036)

Gammie, C.F. \& Popham, R.G., 1998, ApJ, 498, 313

Genzel, R., Eckart, A., Ott, T., \& Eisenhauer, F., 1997, MNRAS, 291, 219

Genzel, R., Hollenbach, D., \& Townes, C. H. 1994, Rep. Prog. Phys., 57, 417

Gruzinov, A. 1998, ApJ, 501, 787

Gruzinov, A. \& Quataert, E., 1998, ApJ submitted astro-ph/9808278)

Haller, J. W., Rieke, M.J., Rieke, G.H., Tamblyn, P., Close, L., \& Melia, F., 1996, 456, 194 (Erratum, 1996 , ApJ, 468, 955)

Hameury, J. M., Lasota, J. P., McClintock, J. E., \& Narayan, R., 1997, ApJ, 489, 234

Hawley, J. F., Gammie, C. F., \& Balbus, S. A., 1996, ApJ, 464, 690

Ichimaru, S. 1977, ApJ, 214, 840

Kato, S., Fukue, J., Mineshige, S., 1998, Black-Hole Accretion Disks (Japan: Kyoto University Press)

Lasota, J. P., Abramowicz, M.A., Chen, X., Krolik, J., Narayan, R., \& Yi, I., 1996a, ApJ, 462, 142

Lasota, J. P., Narayan, R., \& Yi, I., 1996b, A \& A, 314, 813

Mahadevan, R., 1997, ApJ, 477, 585

Mahadevan, R., 1998, Nature, 394, 651

Mahadevan, R, Narayan, R., \& Krolik, J. 1997, ApJ, 486, 268

Mahadevan, R. \& Quataert, E. 1997, ApJ, 490, 605

Manmoto, T., Mineshige, S., \& Kusunose, M., 1997, ApJ, 489, 791

Markoff, S., Melia, F., \& Sarcevic, I., 1997, ApJ, 489, L47

McClintock, J. E., Horne, K., \& Remillard, R. A., 1995, ApJ, 442, 35

Melia, F. 1992, ApJ, 387, L25

Menou, K., Esin, A. A., Narayan, R., Garcia, Lasota, J.P., \& M., McClintock, J. E., 1998, in preparation

Menou, K., Quataert, E., \& Narayan, R., 1998, in Proc. of the 8th Marcel Grossmann Meeting on General Relativity astro-ph/9712015

Menten, K. M., Reid, M. J., Eckart, A., \& Genzel, R. 1997, ApJ, 475, L111

Nakamura, K. E., Masaaki, K., Matsumoto, R., \& Kato, S. 1997, PASJ, 49, 503

Narayan, R. 1996, ApJ, 462, 136

Narayan, R., Barret, D., \& McClintock, J. E., 1997a, ApJ, 482, 448

Narayan, R., Kato, S., \& Honma, F. 1997, ApJ, 476, 49

Narayan, R., Mahadevan, R., Grindlay, J.E., Popham, R.G., \& Gammie, C., 1998a, ApJ, 492, 554

Narayan, R., Mahadevan, R., \& Quataert, E., 1998b, in The Theory of Black Hole Accretion Discs, eds. M.A. 
Abramowicz, G. Bjornsson, and J.E. Pringle (Cambridge: Cambridge University Press)

(astro-ph/9803131)

Narayan, R., McClintock, J.E., \& Yi, I., 1996, ApJ, 457821

Narayan, R., \& Yi, I., 1994, ApJ, 428, L13

Narayan, R., \& Yi, I., 1995, ApJ, 444, 231

Narayan, R., Yi, I., \& Mahadevan, R., 1995, Nature, 374, 623

Peitz, J. \& Appl, S. 1997, MNRAS, 286, 681

Quataert, E. 1998, ApJ, 500, 978

Quataert, E. \& Gruzinov, A., 1998, ApJ submitted (astro-ph/9803112)

Quataert, E. \& Narayan, R., 1998, ApJ submitted (astro-ph/9810117)

Rees, M. J., 1982, in The Galactic Center (ed. G. R. Riegler \& R. D. Blandford). AIP, p. 166, New York Rees, M. J., Begelman, M. C., Blandford, R. D., \& Phinney, E. S., 1982, Nature, 295, 17

Rogers, A. E. E., et al., 1994, ApJ, 434, L59

Serabyn, E., Carlstrom, J., Lay, O., Lid, D.C., Hunter, T.R., \& Lacy, J.H., 1997, ApJ, 490, L77

Shahbaz, T., Ringwald, F.A., Bunn, J.C., Narylor, T., Charles, P.A., \& Casares, J., 1994, MNRAS, 271, L10

Vargas. M. et al., 1996, in The Galactic Center, ed. R. Gredel, 431

Wheeler, J. C., 1996, in Relativistic Astrophysics, eds. B. Jones \& D. Markovic (Cambridge: Cambridge University press) 
Table 1. Model Parameters for V404 Cyg: $r_{\text {out }}=10^{4}, m=12, \dot{M}_{\text {in }} \equiv \dot{M}_{\text {out }} 10^{-4 p}$

\begin{tabular}{|c|c|c|c|c|c|c|c|c|}
\hline Fig. & $\alpha$ & $\beta$ & $\delta$ & $p$ & $\dot{m}_{\text {out }} \times 10^{2}$ & $T_{\mathrm{e}, \max } \times 10^{-10}$ & $L / \dot{M}_{\text {out }} c^{2}$ & $L / \dot{M}_{\text {in }} c^{2}$ \\
\hline $1 \mathrm{a}$ & 0.1 & 10 & 0.01 & 0 & 0.1 & 1.0 & $1.1 \times 10^{-3}$ & $1.1 \times 10^{-3}$ \\
\hline $1 \mathrm{a}$ & 0.1 & 10 & 0.01 & 0.2 & 0.54 & 0.92 & $1.6 \times 10^{-4}$ & $1.0 \times 10^{-3}$ \\
\hline $1 \mathrm{a}$ & 0.1 & 10 & 0.01 & 0.4 & 1.6 & 0.87 & $2.2 \times 10^{-5}$ & $8.8 \times 10^{-4}$ \\
\hline $1 \mathrm{a}$ & 0.1 & 10 & 0.01 & 0.6 & 2.0 & 0.6 & $7.5 \times 10^{-6}$ & $2.0 \times 10^{-3}$ \\
\hline $1 b$ & 0.1 & 1 & 0.01 & 0.4 & 1.2 & 0.57 & $6.0 \times 10^{-5}$ & $2.4 \times 10^{-3}$ \\
\hline $1 b$ & 0.1 & 10 & 0.01 & 0.4 & 1.6 & 0.87 & $2.2 \times 10^{-5}$ & $8.8 \times 10^{-4}$ \\
\hline $1 b$ & 0.1 & 100 & 0.01 & 0.4 & 1.7 & 1.0 & $1.2 \times 10^{-5}$ & $4.8 \times 10^{-4}$ \\
\hline $2 \mathrm{a}$ & 0.3 & 10 & 0.01 & 0.4 & 2.6 & 0.89 & $1.9 \times 10^{-5}$ & $7.6 \times 10^{-4}$ \\
\hline $2 \mathrm{a}$ & 0.1 & 10 & 0.01 & 0.4 & 1.6 & 0.87 & $2.2 \times 10^{-5}$ & $8.8 \times 10^{-4}$ \\
\hline $2 \mathrm{a}$ & 0.03 & 10 & 0.01 & 0.4 & 0.59 & 0.77 & $5.5 \times 10^{-5}$ & $2.2 \times 10^{-3}$ \\
\hline $2 b$ & 0.1 & 10 & $10^{-3}$ & 0.4 & 1.6 & 0.87 & $2.2 \times 10^{-5}$ & $8.8 \times 10^{-4}$ \\
\hline $2 \mathrm{~b}$ & 0.1 & 10 & 0.01 & 0.4 & 1.6 & 0.87 & $2.2 \times 10^{-5}$ & $8.8 \times 10^{-4}$ \\
\hline $2 b$ & 0.1 & 10 & 0.03 & 0.4 & 1.2 & 0.94 & $3.6 \times 10^{-5}$ & $1.4 \times 10^{-3}$ \\
\hline $2 \mathrm{~b}$ & 0.1 & 10 & 0.1 & 0.4 & 1.0 & 1.15 & $6.2 \times 10^{-5}$ & $2.5 \times 10^{-3}$ \\
\hline $2 b$ & 0.1 & 10 & 0.3 & 0.4 & 0.58 & 1.8 & $1.7 \times 10^{-4}$ & $6.8 \times 10^{-3}$ \\
\hline $3 a$ & 0.3 & 1 & 0.01 & 0 & 0.1 & 0.74 & $1.8 \times 10^{-3}$ & $1.8 \times 10^{-3}$ \\
\hline $3 a$ & 0.1 & 10 & 0.01 & 0 & 0.1 & 1.0 & $1.1 \times 10^{-3}$ & $1.1 \times 10^{-3}$ \\
\hline $3 a$ & 0.03 & 30 & 0.01 & 0 & 0.068 & 1.1 & $1.1 \times 10^{-3}$ & $1.1 \times 10^{-3}$ \\
\hline $3 b$ & 0.1 & 10 & 0.01 & 0 & 0.1 & 1.0 & $1.1 \times 10^{-3}$ & $1.1 \times 10^{-3}$ \\
\hline $3 b$ & 0.1 & 10 & 0.1 & 0 & 0.05 & 1.3 & $2.5 \times 10^{-3}$ & $2.5 \times 10^{-3}$ \\
\hline $3 b$ & 0.1 & 10 & 0.3 & 0 & 0.01 & 2.4 & $6.4 \times 10^{-3}$ & $6.4 \times 10^{-3}$ \\
\hline $8 \mathrm{a}$ & 0.1 & 10 & 0.01 & 0 & 0.1 & 1.0 & $1.1 \times 10^{-3}$ & $1.1 \times 10^{-3}$ \\
\hline $8 a$ & 0.1 & 10 & 0.3 & 0.4 & 0.58 & 1.8 & $1.7 \times 10^{-4}$ & $6.8 \times 10^{-3}$ \\
\hline $8 a$ & 0.1 & 10 & 0.75 & 0.8 & 0.64 & 4.8 & $6.3 \times 10^{-5}$ & 0.1 \\
\hline
\end{tabular}


Table 2. Model Parameters for Sgr A*: $r_{\text {out }}=10^{4}, m=2.5 \times 10^{6}, \dot{M}_{\text {in }} \equiv \dot{M}_{\text {out }} 10^{-4 p}$

\begin{tabular}{|c|c|c|c|c|c|c|c|c|}
\hline Fig. & $\alpha$ & $\beta$ & $\delta$ & $p$ & $\dot{m}_{\text {out }} \times 10^{4}$ & $T_{\mathrm{e}, \max } \times 10^{-10}$ & $L / \dot{M}_{\text {out }} c^{2}$ & $L / \dot{M}_{\mathrm{in}} c^{2}$ \\
\hline $4 a$ & 0.1 & 10 & 0.01 & 0 & 0.68 & 2.0 & $2.6 \times 10^{-5}$ & $2.6 \times 10^{-5}$ \\
\hline $4 a$ & 0.1 & 10 & 0.01 & 0.2 & 1.8 & 1.1 & $5.5 \times 10^{-7}$ & $3.5 \times 10^{-6}$ \\
\hline $4 a$ & 0.1 & 10 & 0.01 & 0.4 & 2.4 & 0.63 & $1.2 \times 10^{-7}$ & $4.8 \times 10^{-6}$ \\
\hline $4 a$ & 0.1 & 10 & 0.01 & 0.6 & 2.8 & 0.37 & $8.1 \times 10^{-8}$ & $2.0 \times 10^{-5}$ \\
\hline $4 \mathrm{~b}$ & 0.1 & 1 & 0.01 & 0.4 & 1.9 & 0.47 & $1.2 \times 10^{-7}$ & $4.8 \times 10^{-6}$ \\
\hline $4 \mathrm{~b}$ & 0.1 & 10 & 0.01 & 0.4 & 2.4 & 0.63 & $1.2 \times 10^{-7}$ & $4.8 \times 10^{-6}$ \\
\hline $4 \mathrm{~b}$ & 0.1 & 100 & 0.01 & 0.4 & 2.4 & 0.65 & $1.2 \times 10^{-7}$ & $4.8 \times 10^{-6}$ \\
\hline $5 a$ & 0.3 & 10 & 0.01 & 0.4 & 4.0 & 0.72 & $7.6 \times 10^{-8}$ & $3.1 \times 10^{-6}$ \\
\hline $5 a$ & 0.1 & 10 & 0.01 & 0.4 & 2.4 & 0.63 & $1.2 \times 10^{-7}$ & $4.8 \times 10^{-6}$ \\
\hline $5 a$ & 0.03 & 10 & 0.01 & 0.4 & 1.0 & 0.54 & $2.8 \times 10^{-7}$ & $1.1 \times 10^{-5}$ \\
\hline $5 b$ & 0.1 & 10 & 0.01 & 0.4 & 2.4 & 0.63 & $1.2 \times 10^{-7}$ & $4.8 \times 10^{-6}$ \\
\hline $5 b$ & 0.1 & 10 & 0.1 & 0.4 & 1.7 & 1.6 & $3.1 \times 10^{-7}$ & $1.2 \times 10^{-5}$ \\
\hline $5 b$ & 0.1 & 10 & 0.3 & 0.4 & 1.7 & 3.5 & $3.1 \times 10^{-6}$ & $1.2 \times 10^{-4}$ \\
\hline $6 a$ & 0.3 & 1 & 0.01 & 0 & 0.69 & 1.6 & $7.6 \times 10^{-5}$ & $7.6 \times 10^{-5}$ \\
\hline $6 a$ & 0.1 & 10 & 0.01 & 0 & 0.68 & 2.0 & $2.6 \times 10^{-5}$ & $2.6 \times 10^{-5}$ \\
\hline $6 a$ & 0.03 & 30 & 0.01 & 0 & 0.48 & 1.6 & $7.7 \times 10^{-6}$ & $7.7 \times 10^{-6}$ \\
\hline $6 b$ & 0.1 & 10 & 0.01 & 0 & 0.68 & 2.0 & $2.6 \times 10^{-5}$ & $2.6 \times 10^{-5}$ \\
\hline $6 b$ & 0.1 & 10 & 0.1 & 0 & 0.54 & 2.3 & $2.6 \times 10^{-5}$ & $2.6 \times 10^{-5}$ \\
\hline $6 b$ & 0.1 & 10 & 0.3 & 0 & 0.11 & 5 & $1.0 \times 10^{-4}$ & $1.0 \times 10^{-4}$ \\
\hline $8 b$ & 0.1 & 10 & 0.01 & 0 & 0.68 & 2.0 & $2.6 \times 10^{-5}$ & $2.6 \times 10^{-5}$ \\
\hline $8 b$ & 0.1 & 10 & 0.4 & 0.4 & 1.6 & 4.4 & $6.9 \times 10^{-6}$ & $2.7 \times 10^{-4}$ \\
\hline $8 b$ & 0.1 & 10 & 0.75 & 0.8 & 1.2 & 13.0 & $3.7 \times 10^{-6}$ & $5.9 \times 10^{-3}$ \\
\hline
\end{tabular}


Table 3. Model Parameters for NGC 4649: $r_{\text {out }}=10^{4}, m=8 \times 10^{9}, \dot{M}_{\text {in }} \equiv \dot{M}_{\text {out }} 10^{-4 p}$

\begin{tabular}{ccccccccc}
\hline \hline Fig. & $\alpha$ & $\beta$ & $\delta$ & $p$ & $\dot{m}_{\text {out }}$ & $T_{\mathrm{e}, \max } \times 10^{-10}$ & $L / \dot{M}_{\text {out }} c^{2}$ & $L / \dot{M}_{\text {in }} c^{2}$ \\
\hline $7 \mathrm{a}$ & 0.1 & 10 & 0.01 & 0 & $10^{-3}$ & 1.9 & $3.1 \times 10^{-4}$ & $3.1 \times 10^{-4}$ \\
$7 \mathrm{a}$ & 0.1 & 10 & 0.3 & 0.25 & $10^{-3}$ & 3.4 & $3.6 \times 10^{-5}$ & $3.6 \times 10^{-4}$ \\
$7 \mathrm{a}$ & 0.1 & 10 & 0.01 & 0.25 & $10^{-3}$ & 1.0 & $2.1 \times 10^{-6}$ & $2.1 \times 10^{-5}$ \\
$7 \mathrm{a}$ & 0.1 & 10 & 0.3 & 0.54 & $10^{-3}$ & 2.4 & $6.6 \times 10^{-7}$ & $9.5 \times 10^{-5}$ \\
& & & & & & & & \\
$7 \mathrm{~b}$ & 0.1 & 10 & 0.01 & 0 & $10^{-4.5}$ & 1.9 & $2.6 \times 10^{-6}$ & $2.6 \times 10^{-6}$ \\
$7 \mathrm{~b}$ & 0.1 & 10 & 0.3 & 0.25 & $10^{-4.5}$ & 3.5 & $9.1 \times 10^{-7}$ & $9.1 \times 10^{-6}$ \\
$7 \mathrm{~b}$ & 0.1 & 10 & 0.01 & 0.25 & $10^{-4.5}$ & 1.0 & $5.7 \times 10^{-8}$ & $5.7 \times 10^{-7}$ \\
\hline
\end{tabular}
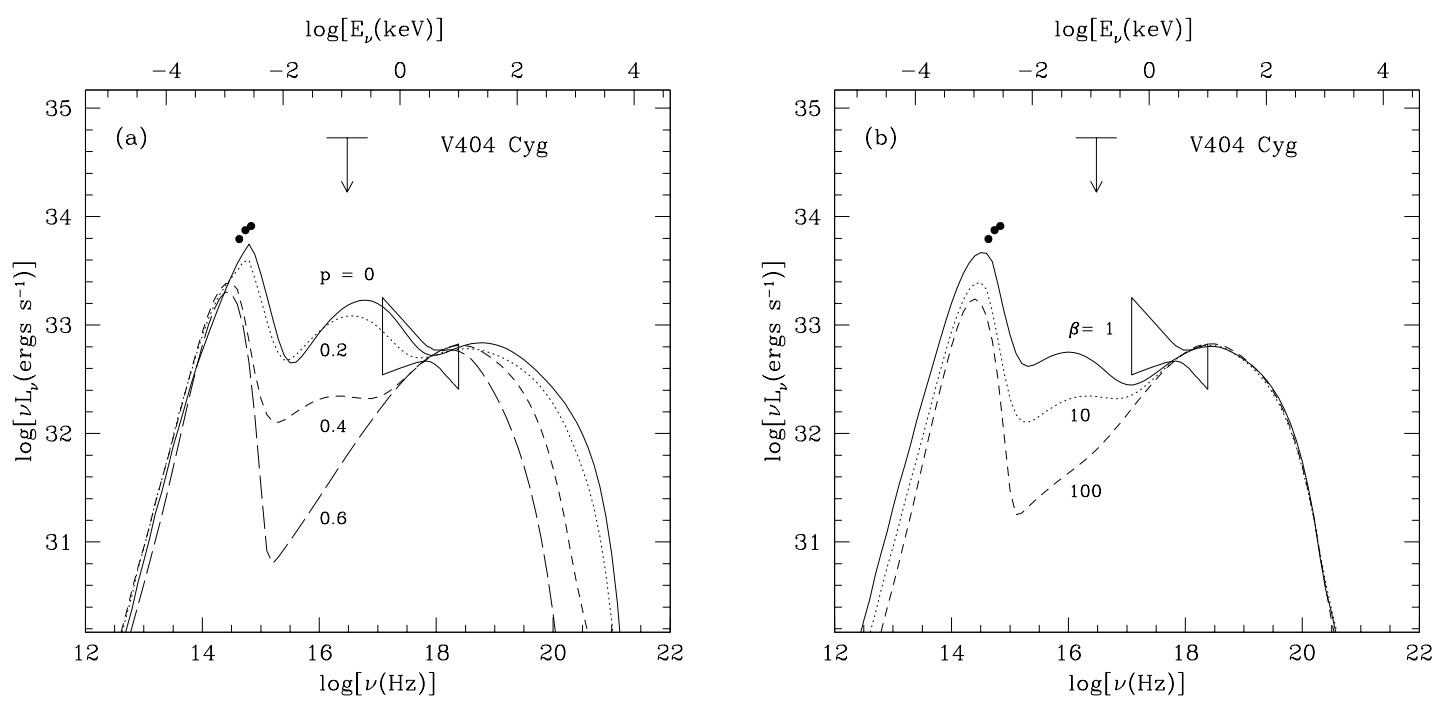

Fig. 1.- (a) Spectral models of V404 Cyg for several values of $p$, taking $\alpha=0.1, \beta=10$, and $\delta=0.01$. (b) Models for several $\beta$, taking $\alpha=0.1, p=0.4$, and $\delta=0.01$. 

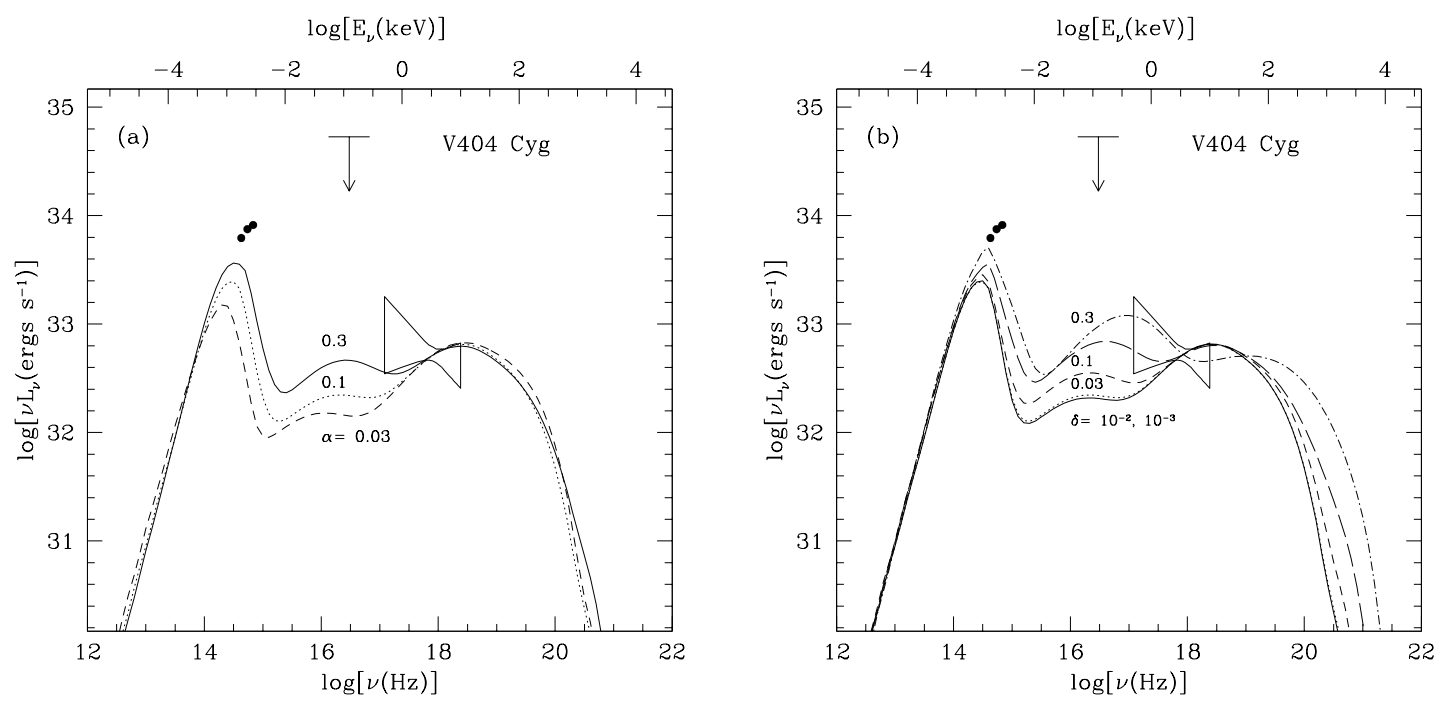

Fig. 2.- (a) Spectral models of V404 Cyg for several values of $\alpha$, taking $p=0.4, \beta=10$, and $\delta=0.01$. (b) Models for several $\delta$, taking $\alpha=0.1, p=0.4, \beta=10$.
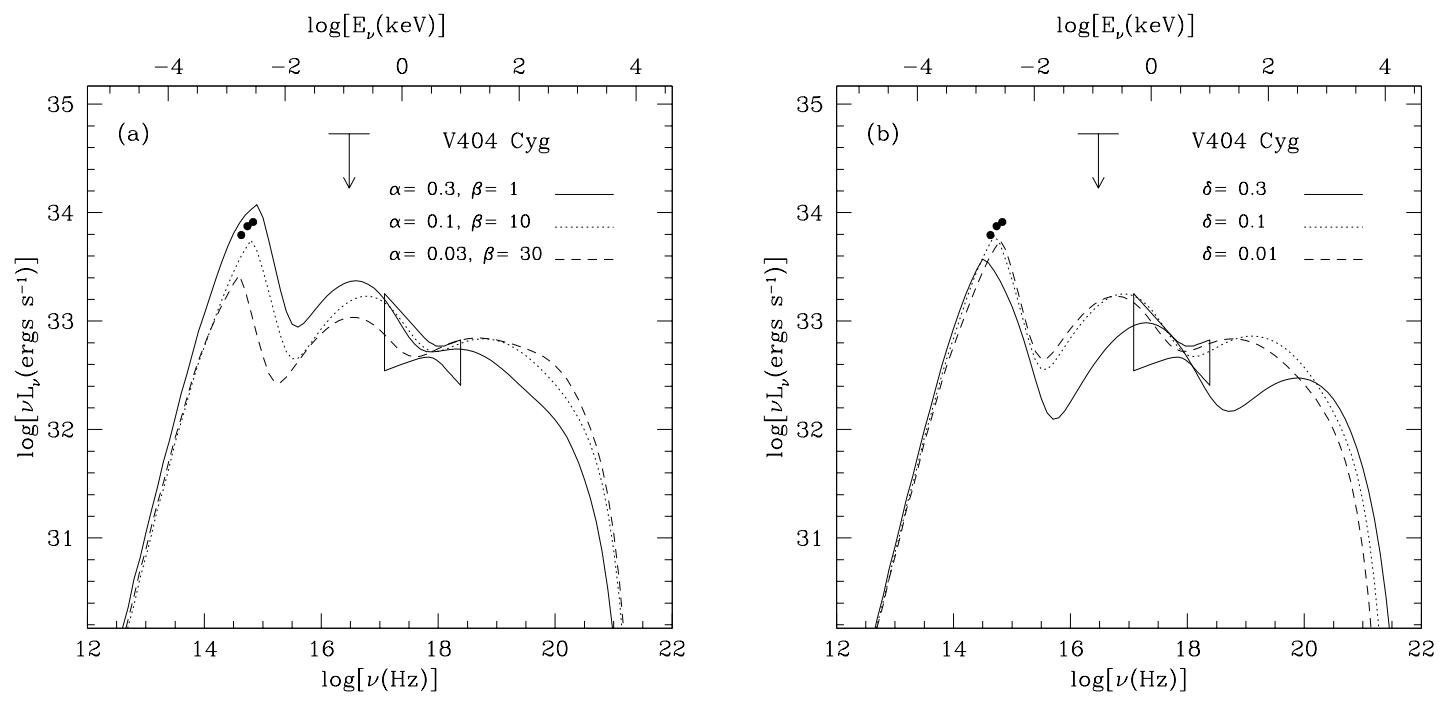

Fig. 3.- (a) No wind $(p=0)$ spectral models of V404 Cyg for several values of $\alpha$ and $\beta$, taking $\delta=0.01$. (b) Models for several values of $\delta$, taking $p=0, \alpha=0.1$, and $\beta=10$. 

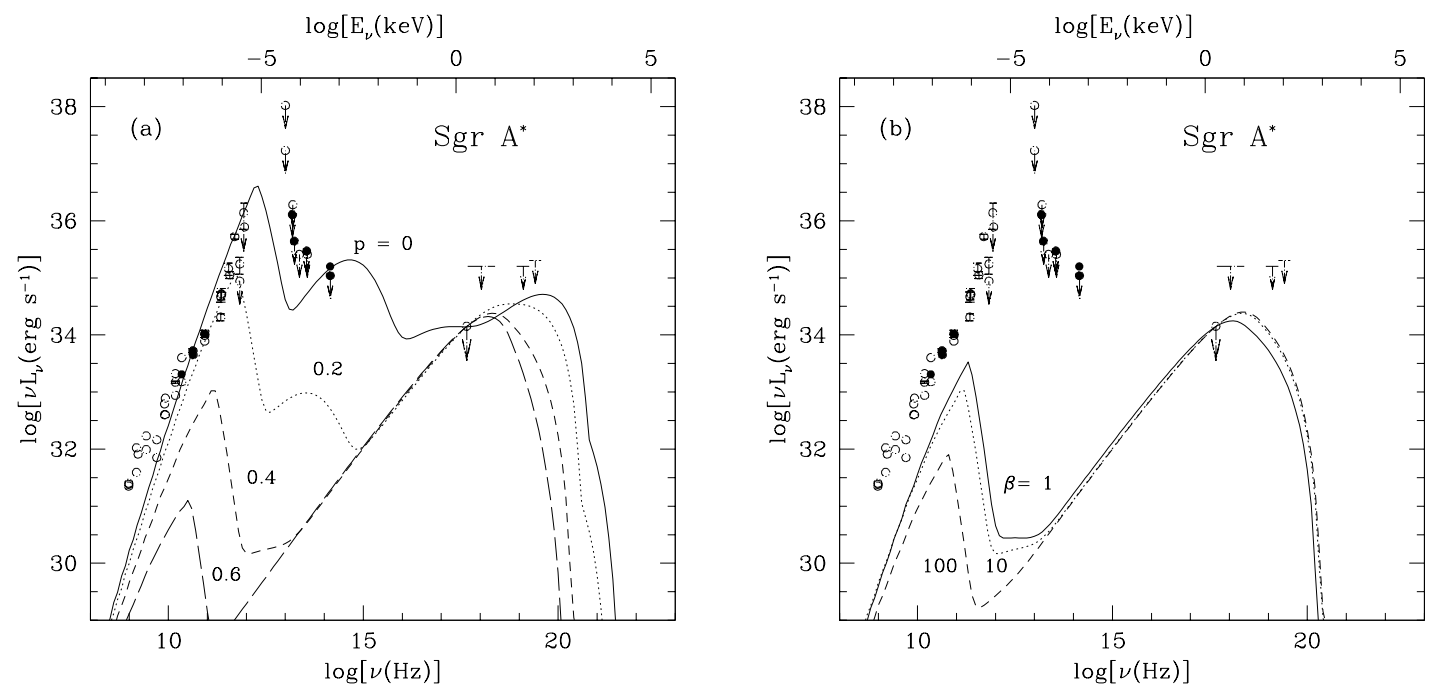

Fig. 4.- (a) Spectral models of Sgr A* for several values of $p$, taking $\alpha=0.1, \beta=10$, and $\delta=0.01$. (b) Models for several $\beta$, taking $\alpha=0.1, p=0.4$, and $\delta=0.01$.
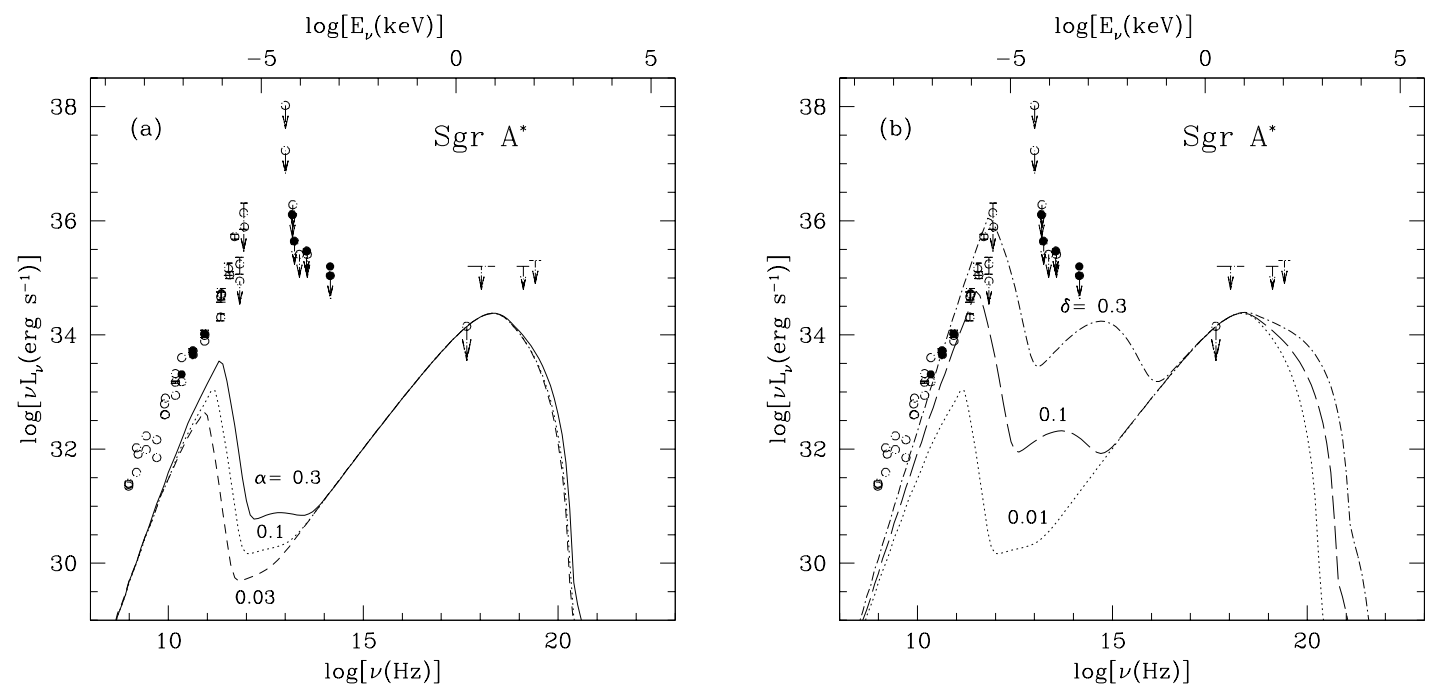

Fig. 5.- (a) Spectral models of Sgr A* for several values of $\alpha$, taking $p=0.4, \beta=10$, and $\delta=0.01$. (b) Models for several $\delta$, taking $\alpha=0.1, p=0.4, \beta=10$. 

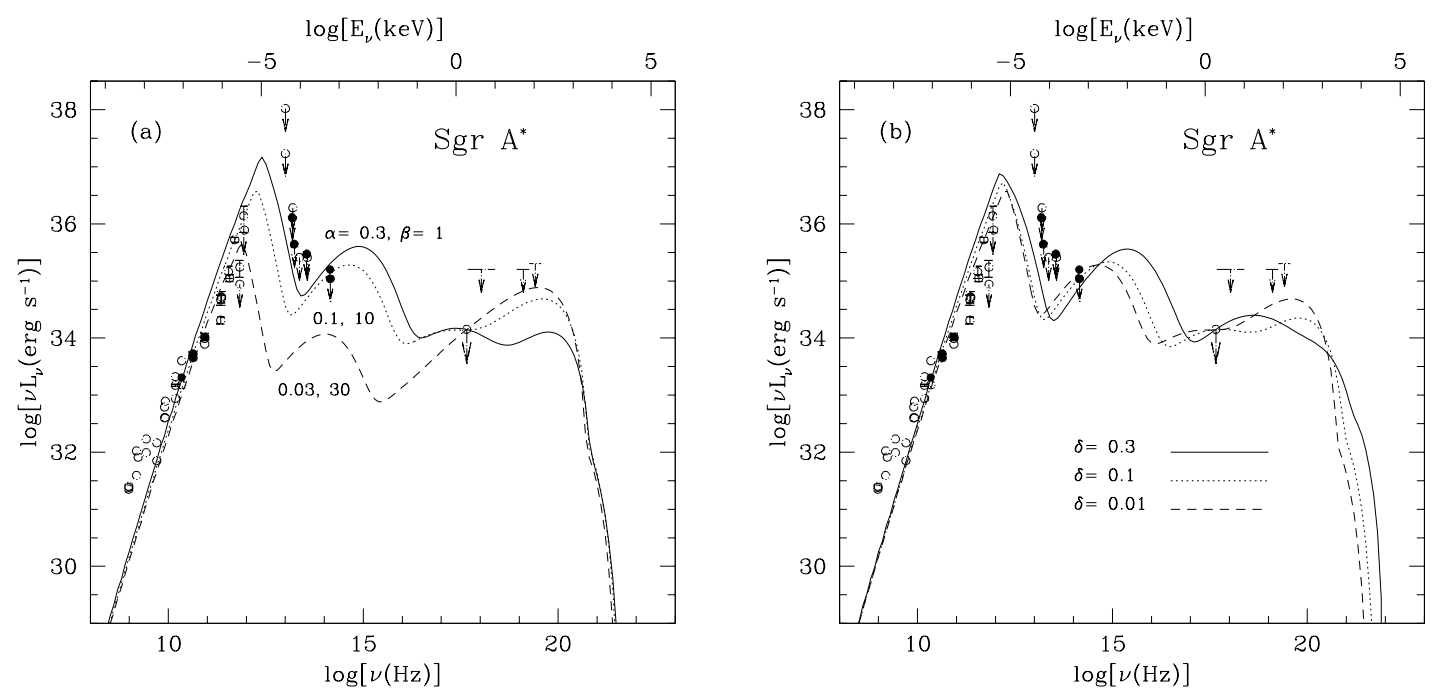

Fig. 6.- (a) Spectral models of Sgr A* for several values of $\alpha$ and $\beta$, taking $p=0, \delta=0.01$. (b) Models for several $\delta$, taking $\alpha=0.1, \beta=10$, and $p=0$.
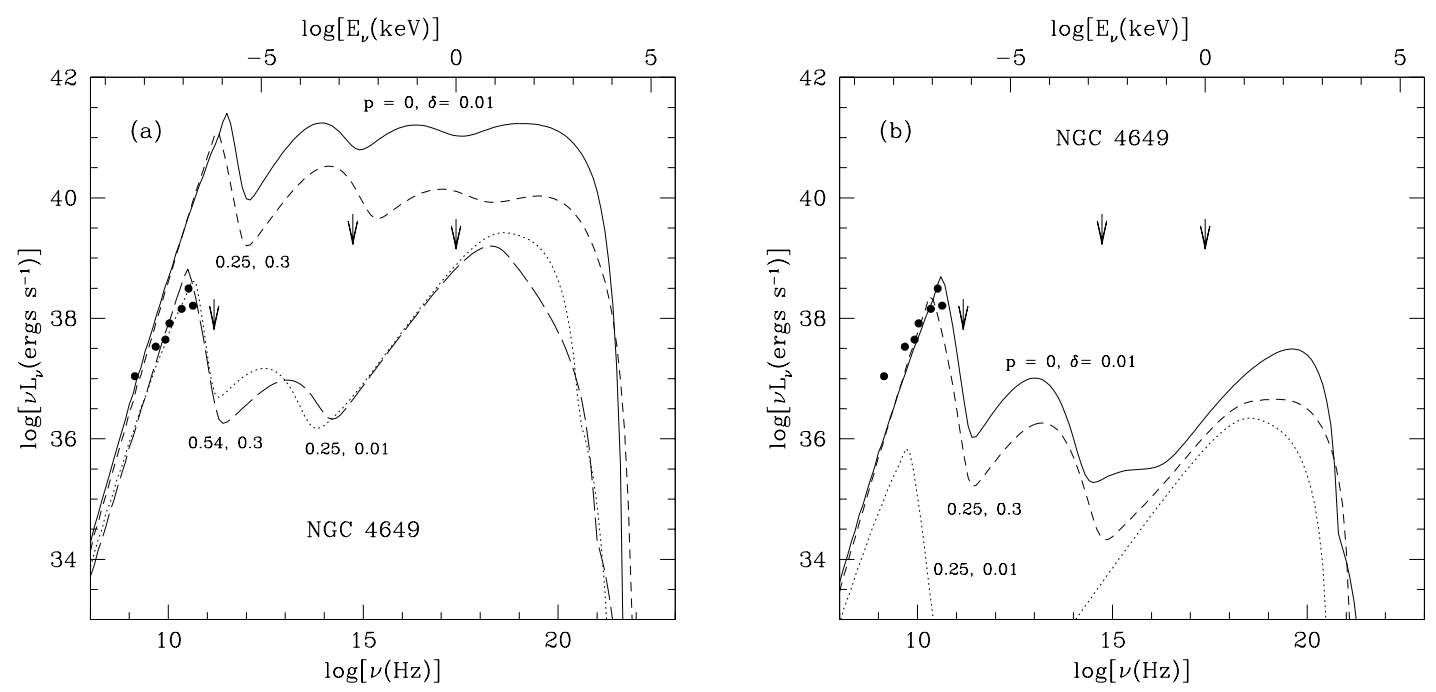

Fig. 7.- Spectral models of NGC 4649 for several values of $p$ and $\delta$, taking $\alpha=0.1, \beta=10$, $r_{\text {out }}=10^{4}$, and $m=8 \times 10^{9}$. Panel (a) assumes that the accretion rate at the outer edge of the flow is $\dot{m}_{\text {out }}=10^{-3}$, while panel (b) takes $\dot{m}_{\text {out }}=10^{-4.5}$. 

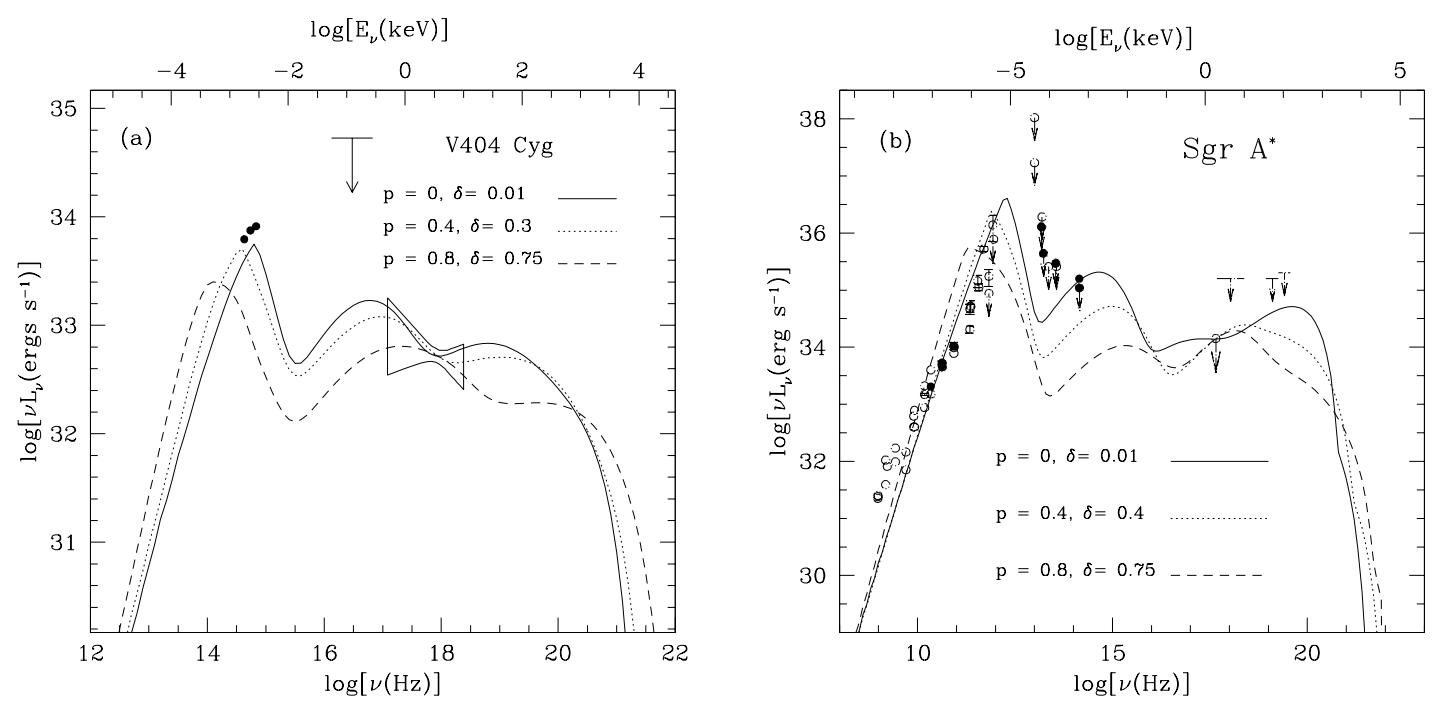

Fig. 8. - Spectral models of V404 Cyg (Fig. 8a) and Sgr A* (Fig. 8b) for several values of $p$ and $\delta$, taking $\alpha=0.1, \beta=10$, and $r_{\text {out }}=10^{4}$. 